\title{
Density dependence of the symmetry energy from neutron skin thickness in finite nuclei
}

\author{
X. Viñas ${ }^{1}$, M. Centelles ${ }^{1}$, X. Roca-Maza ${ }^{2}$, and M. Warda ${ }^{3}$ \\ 1 Departament d'Estructura i Constituents de la Matèria and Institut de Ciències del Cosmos, Facultat de Física, Universitat \\ de Barcelona, Diagonal 645, E-08028 Barcelona, Spain \\ 2 Dipartamento di Fisica, Università degli Studi di Milano and INFN, Via Celoria 16, I-20133 Milano, Italy \\ 3 Katedra Fizyki Teoretycznej, Uniwersytet Marii Curie-Skłodowskiej ul. Radziszewskiego 10, 20-031 Lublin, Poland
}

Received: date / Revised version: date

\begin{abstract}
The density dependence of the symmetry energy around saturation density, characterized by the slope parameter $L$, is studied using information provided by the neutron skin thickness in finite nuclei. An estimate for $L$ is obtained from experimental data on neutron skins extracted from antiprotonic atoms. We also discuss the ability of parity-violating elastic electron scattering to obtain information on the neutron skin thickness in ${ }^{208} \mathrm{~Pb}$ and to constrain the density dependence of the nuclear symmetry energy. The size and shape of the neutron density distribution of ${ }^{208} \mathrm{~Pb}$ predicted by mean-field models is briefly addressed. We conclude with a comparative overview of the $L$ values predicted by several existing determinations.
\end{abstract}

PACS. 21.10.Gv, 21.65.Ef, 21.60.-n, 25.30.Bf, 26.60.-c Symmetry energy. Neutron density. Neutron skin thickness. Parity-violating electron scattering.

\section{Introduction}

The nuclear symmetry energy is a fundamental quantity in nuclear physics and astrophysics because it governs important properties of very small entities like atomic nuclei $\left(R \sim 10^{-15} \mathrm{~m}\right)$ and of very large objects like neutron stars $\left(R \sim 10^{4} \mathrm{~m}\right)[1$. The symmetry energy represents the energy cost per particle of changing all the protons into neutrons in symmetric nuclear matter. In nuclear physics the symmetry energy has important consequences for studies of the neutron distribution in stable and exotic nuclei 2 9, heavy-ion collisions (HIC) 10 16, giant and pygmy resonances [17 21, etc. The symmetry energy is also relevant for some aspects of fundamental physics because it has implications for atomic parity non-conserving observables involved in low-energy tests of the Standard Model and new physics [22, 23. In astrophysics the symmetry energy is very important for understanding different properties of neutron stars, supernova explosions, and stellar nucleosynthesis [1, 24, 26].

The Equation of State (EOS) in asymmetric nuclear matter can be expressed as

$$
e(\rho, \delta)=e(\rho, 0)+S(\rho) \delta^{2}+\mathcal{O}\left(\delta^{4}\right)
$$

where $\rho=\rho_{n}+\rho_{p}$ is the total nucleon density and $\delta=$ $\left(\rho_{n}-\rho_{p}\right) / \rho$ defines the relative asymmetry parameter. In Eq. (1), $e(\rho, \delta)$ is the energy per particle in asymmetric nuclear matter and $S(\rho)$ is the symmetry energy which is defined as

$$
S(\rho)=\left.\frac{1}{2} \frac{\partial^{2} e(\rho, \delta)}{\partial \delta^{2}}\right|_{\delta=0} .
$$

To a good accuracy $S(\rho)$ can be estimated as the difference between the EOS of pure neutron matter and symmetric nuclear matter, i.e.,

$$
S(\rho) \approx e(\rho, \delta=1)-e(\rho, \delta=0) .
$$

It is both common and insightful to expand the EOS of asymmetric nuclear matter around the saturation density $\rho_{0}$ using a few meaningful bulk parameters, as in

$$
e(\rho, \delta=0) \simeq a_{v}+\frac{1}{2} K_{v} \epsilon^{2}
$$

and

$$
S(\rho) \simeq J-L \epsilon+\frac{1}{2} K_{\mathrm{sym}} \epsilon^{2},
$$

where the density shift is defined as $\epsilon=\left(\rho_{0}-\rho\right) /\left(3 \rho_{0}\right)$. The energy per particle $a_{v}$ and incompressibility $K_{v}$ in symmetric nuclear matter at saturation density $\rho_{0} \approx 0.16$ $\mathrm{fm}^{-3}$ are well constrained by the measured data on densities, masses and collective excitations of nuclei. They are around $a_{v} \approx-16 \mathrm{MeV}$ and $K_{v} \approx 230 \pm 30 \mathrm{MeV}$ 27. The symmetry energy coefficient is defined as $J=S\left(\rho_{0}\right)$. Experimental informations from nuclear masses, heavy-ion reactions, giant resonances, and observational properties of neutron stars, constrain $J$ in a range between 30 and $35 \mathrm{MeV}$ 28, 29. These values are, in general, predicted also by successful nuclear mean-field models. 

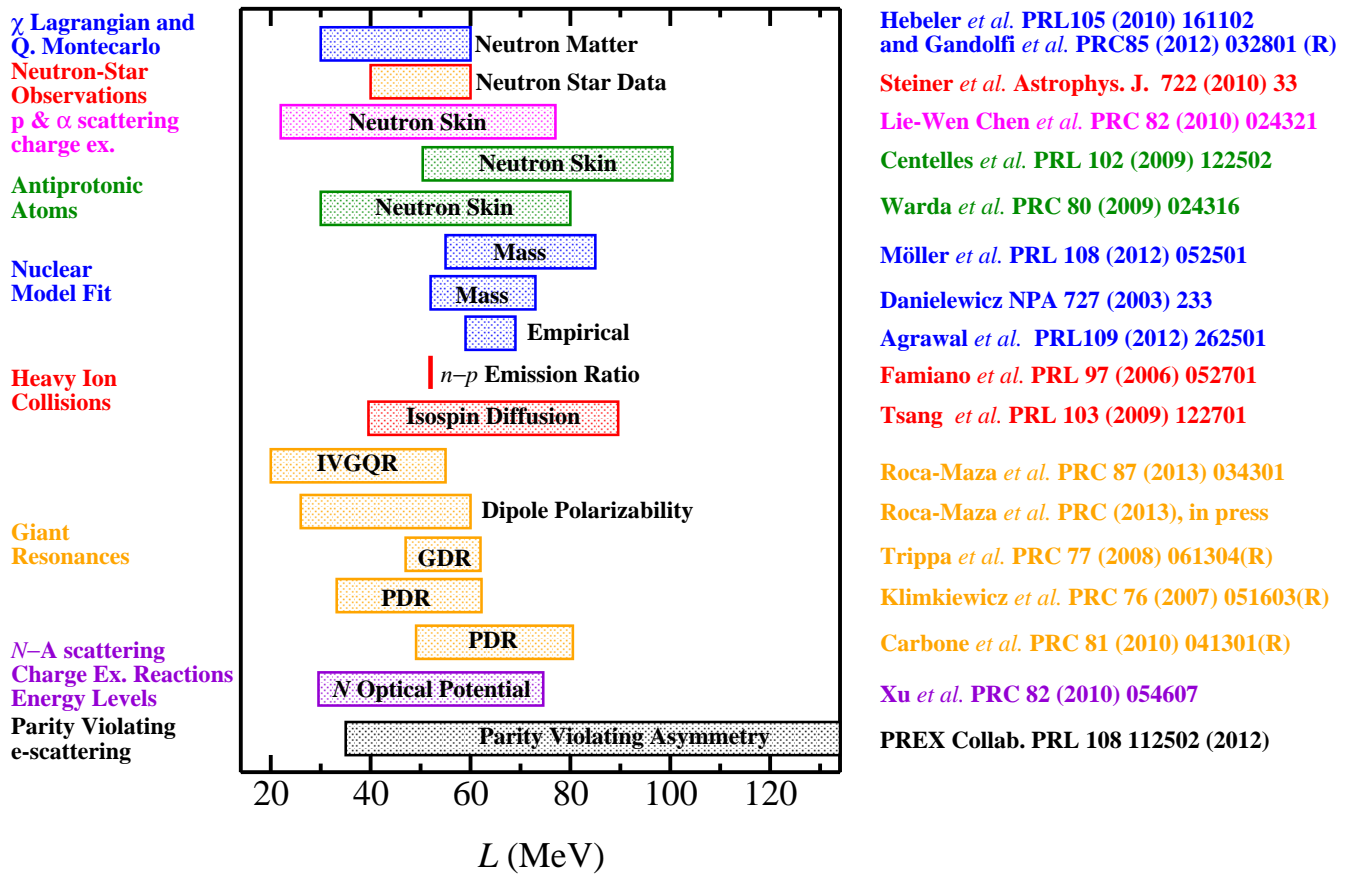

Fig. 1. (Color online) Different available estimates of the slope parameter of the symmetry energy $L$.

However, the density dependence of the symmetry energy, characterized by the parameters

$$
L=\left.3 \rho \frac{\partial S(\rho)}{\partial \rho}\right|_{\rho_{0}}
$$

and

$$
K_{\text {sym }}=\left.9 \rho^{2} \frac{\partial^{2} S(\rho)}{\partial \rho^{2}}\right|_{\rho_{0}},
$$

is much less certain and the predicted values differ largely among different nuclear models (see e.g. Ref. [13]). This is a consequence of the fact that data of stable nuclei, used in the fits of the parameters of the models, do not constrain in a very precise way the isovector sector of the effective nuclear interaction. The $L$ value predicted by successful mean-field models covers a wide range roughly between 20 $\mathrm{MeV}$ and $120 \mathrm{MeV}$. To extract this quantity from available experimental data is not an easy task and usually some model assumptions have to be made in order to connect the data with the parameter $L$.

A sample of different experimental results and theoretical calculations used to estimate $L$ is displayed in Fig. 1 . We will overview them in some more detail later in Section 5. For in-depth accounts of several of the methods and determinations shown in Fig. 1. see the related contributions in the present volume (also see Refs. [28, 29]). Fig. 1 illustrates that although very different observables and methods have been used to extract $L$ and different values have been obtained, there is a common range, approximately between 45 and $70 \mathrm{MeV}$, where the different estimates of $L$ have a large overlap. Such a range can be considered as a reliable estimate of the $L$ parameter. This range of values agrees also with the range reported in Ref. [27.
On the basis of our findings in recent years [30 34, in the next sections we will discuss two of these estimates of the $L$ parameter. Namely, we will study the constraints that can be derived from the analysis of neutron skins in antiprotonic atoms, and the constraints that may be provided by parity-violating elastic electron scattering in a heavy neutron-rich nucleus such as ${ }^{208} \mathrm{~Pb}$. We also discuss the size and shape of the neutron density distribution of ${ }^{208} \mathrm{~Pb}$ predicted by mean-field models and its dependence with the symmetry energy. A summary and outlook are given to conclude the paper.

\section{Insights into the neutron skin thickness from the nuclear droplet model}

\subsection{Theory}

The neutron skin thickness (NST) of a nucleus is conventionally defined as the difference between the neutron and proton rms radii of the nucleus:

$$
\Delta r_{n p}=\left\langle r^{2}\right\rangle_{n}^{1 / 2}-\left\langle r^{2}\right\rangle_{p}^{1 / 2}
$$

It is known from almost fifteen years ago that in a heavy nucleus such as ${ }^{208} \mathrm{~Pb}$ the NST calculated with successful mean-field models that reproduce the binding energy and charge radius of this nucleus is linearly correlated with the slope of the symmetry energy at saturation computed with the same model [2 4]. This correlation can be clearly seen in Fig. 2, where Hartree-Fock or Hartree calculations of the NST of ${ }^{208} \mathrm{~Pb}$ with different Skyrme, Gogny and relativistic mean-field models is displayed as a function of 


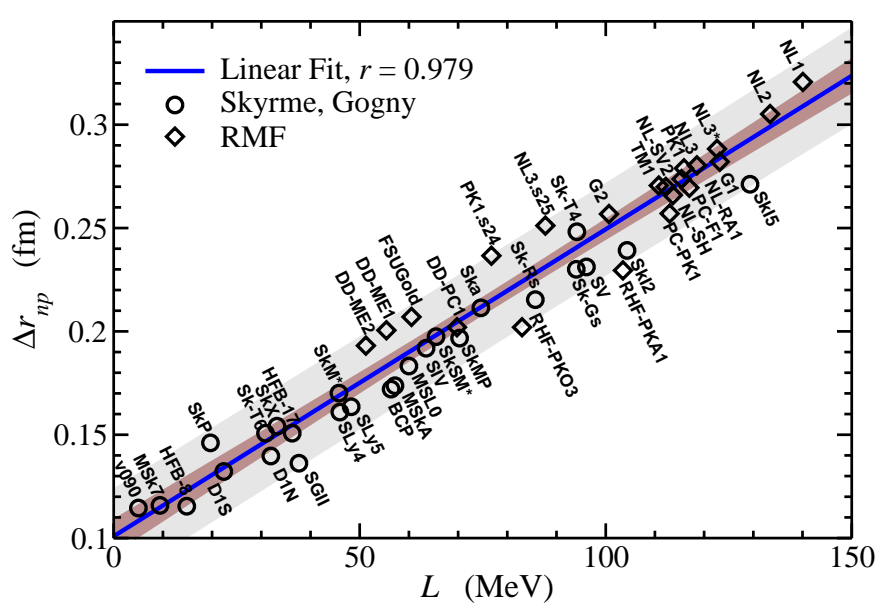

Fig. 2. (Color online) Neutron skin thickness $\Delta r_{n p}$ of ${ }^{208} \mathrm{~Pb}$ against the slope parameter of the symmetry energy at saturation density $L$. The predictions of diverse nuclear energy density functionals (including Skyrme and Gogny forces and relativistic mean-field models) are shown. The linear correlation coefficient of the results for $\Delta r_{n p}$ and $L$ is $r=0.979$. A linear fit gives $\Delta r_{n p}=0.101+0.00147 L$, with $\Delta r_{n p}$ in $\mathrm{fm}$ and $L$ in $\mathrm{MeV}$. The thinner and thicker shadowed regions represent, respectively, the $95 \%$-confidence band and the $95 \%$ prediction band of the linear regression [35. Figure adapted from Ref. 34.

the parameter $L$ defined in Eq. (6), which is proportional to the slope of the symmetry energy at saturation. The linear fit of the results of Fig. 2 gives

$$
\Delta r_{n p}(\mathrm{fm})=0.101+0.00147 L(\mathrm{MeV}) .
$$

Information about the models of the figure is provided in Section 3.2

A possible explanation of the correlation between $\Delta r_{n p}$ and $L$ on the basis of the nuclear Droplet Model (DM) 36 38 has been reported in Ref. 30. In the DM, the symmetry energy coefficient of a nucleus with mass number $A$ is obtained as $36-38$

$$
a_{\mathrm{sym}}^{\mathrm{DM}}(A)=\frac{J}{1+x_{A}}, \quad \text { with } x_{A}=\frac{9 J}{4 Q} A^{-1 / 3},
$$

where $J$ is the bulk symmetry energy coefficient defined previously. The corresponding symmetry energy contribution to a finite nucleus is given by 36,38 .

$$
E_{\mathrm{sym}}^{\mathrm{DM}}(A)=a_{\mathrm{sym}}^{\mathrm{DM}}(A)\left(I+x_{A} I_{C}\right)^{2} A,
$$

where $I=(N-Z) / A$ is the overall relative neutron excess. The quantity $I_{\mathrm{C}}=e^{2} Z /(20 J R)$, where $R=r_{0} A^{1 / 3}$ is the nuclear radius, is a correction due to the Coulomb interaction 36 38.

The coefficient $Q$ of Eq. 10 is called the surface stiffness coefficient. It measures the resistance of neutrons against separation from protons to form the neutron skin. In the context of the DM and under the assumption that a finite nucleus is a leptodermous finite Fermi system, having a wide bulk part and a narrow surface region where the
Table 1. Coefficients $J$ and $a_{\mathrm{sym}}^{\mathrm{DM}}(A)$ and density $\rho$ that fulfils $S(\rho)=a_{\mathrm{sym}}^{\mathrm{DM}}(A)$ for $A=208$ in several nuclear models. The values of $a_{\mathrm{sym}}^{\mathrm{DM}}(A)$ and $S(\rho)$ have been computed with Eqs. (10) and 2), respectively.

\begin{tabular}{lccc}
\hline Model & $J(\mathrm{MeV})$ & $a_{\text {sym }}^{\text {DM }}(\mathrm{MeV})$ & $\rho\left(\mathrm{fm}^{-3}\right)$ \\
\hline NL3 & 37.4 & 25.8 & 0.103 \\
NL-SH & 36.1 & 26.0 & 0.105 \\
FSUGold & 32.6 & 25.4 & 0.099 \\
TF & 32.6 & 24.2 & 0.094 \\
SLy4 & 32.0 & 25.3 & 0.100 \\
SkX & 31.1 & 25.7 & 0.103 \\
SkM & 30.0 & 23.2 & 0.101 \\
SIII & 28.2 & 24.1 & 0.093 \\
SGII & 26.8 & 21.6 & 0.104 \\
\hline
\end{tabular}

density rapidly drops to zero, nuclear surface properties such as e.g. the coefficient $Q$ can be extracted from semiinfinite nuclear matter calculations 36,42 . The semi-infinite geometry is free of finite-size effects, Coulomb, and shell effects. Quantal or semiclassical extended Thomas-Fermi (ETF) calculations of asymmetric semi-infinite nuclear matter (ASINM) can be performed to compute $Q$ 39 44. Here we perform ETF calculations of ASINM, since the semiclassical ETF method does not present Friedel oscillations found in the densities of a Hartree-Fock or Hartree calculation of ASINM [45, 46. A semiclassical approach does not have quantal shell effects and hence is consistent with the DM concept of an average description of nuclear properties 40. Details of our ETF calculations are given in 43 and in the appendix of 31. For a comparison of some DM predictions against self-consistent ETF results in finite nuclei, see Section 2.3 below.

A link between finite nuclei observables, described by the DM, and certain quantities such as $J$ and $L$ that define the EOS in nuclear matter around saturation, may be obtained from the following empirical consideration [30, 31]. The DM symmetry energy coefficient $a_{\mathrm{sym}}^{\mathrm{DM}}(A)$ of a heavy nucleus like ${ }^{208} \mathrm{~Pb}$ equals the value of the symmetry energy in infinite matter $S(\rho)$ at a subsaturation density near $0.1 \mathrm{fm}^{-3}$ when computed in mean-field models. More recently, this relation has been discussed by Liu et al. from measured nuclear masses 47, by Chen et al. in the Skyrme-Hartree-Fock theory 48, 49, and by Dong et al. in a variety of mean-field interactions [50, 51. For several nuclear models, we display in Table 1 the bulk symmetry energy coefficient $J$, the DM symmetry energy coefficient 10 , and the value of the density $\rho$ that fulfils $S(\rho)=a_{\mathrm{sym}}^{\mathrm{DM}}(A)$ for $A=208$. Of course, though it is around $0.1 \mathrm{fm}^{-3}$, there is a certain variation in the value of $\rho$ where the condition is fullfilled exactly.

Within the DM the NST of a nucleus is given by 52 ]

$$
\Delta r_{n p}^{\mathrm{DM}}=\sqrt{\frac{3}{5}}\left[t-\frac{e^{2} Z}{70 J}+\frac{5}{2 r_{0} A^{1 / 3}}\left(b_{n}^{2}-b_{p}^{2}\right)\right] .
$$

The quantity $t$ is the distance between the neutron and proton mean surface locations. The correction $e^{2} Z / 70 J$ is 
due to the Coulomb interaction, and $b_{n}$ and $b_{p}$ are the surface widths of the neutron and proton density profiles. Eq. (12) shows that the NST in a heavy nucleus may be formed by two basic mechanisms. One of them is due to the separation between the neutron and proton mean surfaces, which is a bulk effect. The other way to form the NST is a surface effect due to the fact that the widths of the neutron and proton surfaces may be different.

In the DM the bulk contribution $t$ to the NST can be written as

$$
\begin{aligned}
t & =\frac{3}{2} r_{0} \frac{J}{Q} \frac{1}{1+x_{A}}\left(I-I_{C}\right) \\
& =\frac{2 r_{0}}{3 J}\left[J-a_{\mathrm{sym}}^{\mathrm{DM}}(A)\right] A^{1 / 3}\left(I-I_{C}\right),
\end{aligned}
$$

where use is made of Eq. 10 to establish the second equality. From the first equality in (13), it is seen that the leading contribution to $t$ in a large nucleus is the term $\frac{3}{2} r_{0}(J / Q) I$. Thus, the DM suggests a correlation between $\Delta r_{n p}$ and $J / Q$ in heavy nuclei. The second equality in 13 shows that the DM establishes a direct relation between the surface symmetry energy coefficient $\left[J-a_{\mathrm{sym}}^{\mathrm{DM}}(A)\right] A^{1 / 3}$ and the bulk part of the NST $t$. The standard version of the DM assumes $b_{n}=b_{p}=1 \mathrm{fm}$ [36 38]. Taking into account the relation between the symmetry energy in infinite matter and the symmetry energy coefficient of a finite nucleus in the DM model $\left(S(\rho) \approx a_{\mathrm{sym}}^{\mathrm{DM}}(A)\right.$ at a subsaturation density $\rho$ ), we can write

$$
t=\frac{2 r_{0}}{3 J} L\left(1-\epsilon \frac{K_{\mathrm{sym}}}{2 L}\right) \epsilon A^{1 / 3}\left(I-I_{C}\right) .
$$

The result explicitly shows the imprint of the density dependence of the symmetry energy on the NST. The leading proportionality in Eq. 14 between the bulk part of the NST $t$ and the slope parameter of the symmetry energy $L$, explains the observed correlation of $\Delta r_{n p}$ with $L$ in a heavy nucleus such as ${ }^{208} \mathrm{~Pb}$, 2, 4, 30, displayed in Fig. 2. Regarding Eq. (14) it should be pointed out that the corrective term with $K_{\text {sym }}$ has a limited influence on the result as far as $\epsilon$ is small.

\subsection{Constraints from empirical data on the neutron skin thickness from antiprotonic atoms}

Slow-enough antiprotons can form with an atomic nucleus a hydrogen-like atom. When the antiproton annihilates with a nucleon, pions are produced that may miss the nucleus and a residue is left with a neutron or proton less. From the analysis of the yields, information about the neutron distribution in the nucleus can be derived (cf. [53, 54 and references therein). A second method measures antiprotonic X-rays, which allow to find the atomic level widths and shifts due to the strong interaction 53 55. Combining the results from the two techniques, the difference $\Delta r_{n p}$ between the neutron and proton rms radii can be estimated if the charge density of the nucleus is known 53 55. The NST of 26 stable nuclei, ranging from

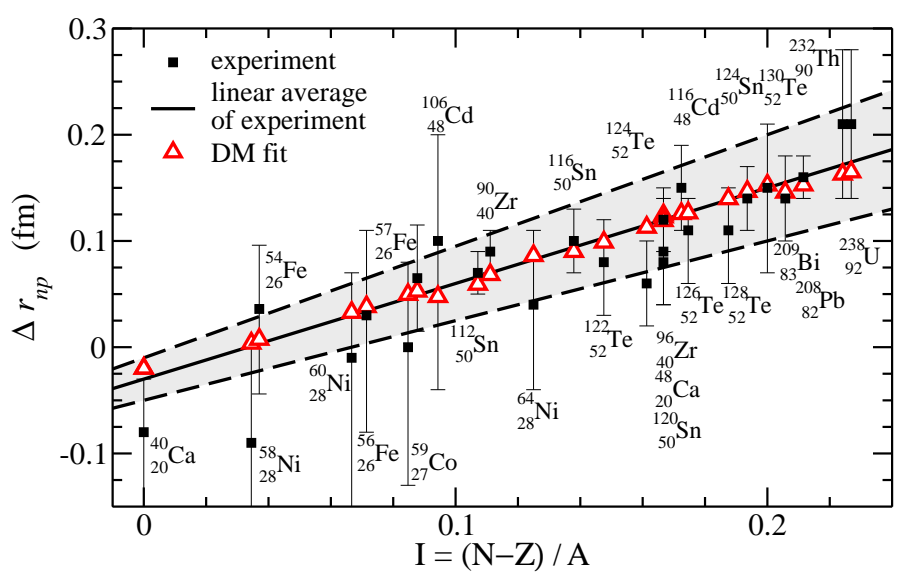

Fig. 3. (Color online) Neutron skin thickness as a function of the asymmetry parameter of the nucleus $I=(N-Z) / A$ for the nuclei measured in the experiments with antiprotonic atoms. The droplet model results have been obtained with Eqs. (12) and (13) (see text for details). Figure adapted from Ref. 30.

${ }^{40} \mathrm{Ca}$ to ${ }^{238} \mathrm{U}$, was extracted from experiments with antiprotons at CERN by Trzcińska et al. 53, 54. The data roughly follow a linear trend with the relative neutron excess $I$ of the nucleus that can be fitted by [53, 54]

$$
\Delta r_{n p}=(0.90 \pm 0.15) I+(-0.03 \pm 0.02) \mathrm{fm} .
$$

It is to be mentioned that the NST values derived from antiprotonic atoms have relatively large uncertainties, as it can be seen in Fig. 2, and sometimes the results deduced from different experiments are not completely concordant with each other [54, 56]. However, the NST data of Refs. [53, 54] are the largest available set of $\Delta r_{n p}$ values uniformly measured with the same experimental technique all over the periodic table $(40 \leq A \leq 238)$. Moreover, all of the nuclei are stable isotopes.

We next explore 30 the constraints on the density dependence of the symmetry energy by fitting Eq. (12) to the neutron skins measured in Refs. [53, 54]. To have a large set of skins across the mass table at our avail is very helpful because the result of the fit is driven mainly by the average trend and is less contingent on the data uncertainties. In our fit the explicit dependence of the NST on $L$ is provided by Eq. (14). In this analysis we parametrize the symmetry energy as

$$
S(\rho)=J\left(\rho / \rho_{0}\right)^{\gamma},
$$

with $J$ the symmetry energy coefficient. This expression has been often used in studies of HIC assuming $J=31.6$ $\mathrm{MeV}$ [13 15, 57, 58]. To start with, we set $b_{n}=b_{p}$ as has been done in the standard DM [36, 38, 52, 59] and in the analysis of data in Ref. 60. In this way we find $L=75 \pm 25 \mathrm{MeV}(\gamma=0.79 \pm 0.25)$ from the fit. The quoted uncertainty is from the window of the linear averages of experiment. The $L$ value and its uncertainty obtained from the DM NST formula with a vanishing surface width correction is compatible with the $L$ values predicted from isospin diffusion and isoscaling observables in HIC in 


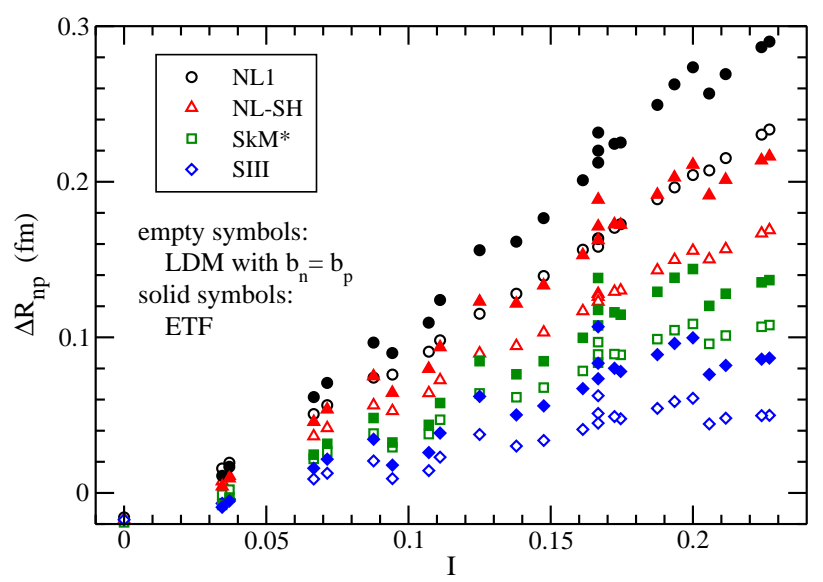

Fig. 4. (Color online) The neutron skin thickness predicted by the standard version of the DM (Eq. 12 with $\left.b_{n}=b_{p}\right)$ is compared with the result from self-consistent ETF calculations of finite nuclei, in four mean-field models. The nuclei considered are those investigated in antiprotonic atoms in Refs. [53, 54 and have masses $40 \leq A \leq 238$. Figure taken from Ref. 31.

Refs. 13, 14, 57, 58. In Fig. 3 we display by open triangles the NST predicted by our DM calculation for the 26 nuclei; the results nicely reproduce the average trend of the experimental data.

\subsection{Contribution of the nuclear surface to the neutron skin thickness}

The results just discussed have been obtained assuming same neutron and proton surface widths (standard version of the DM). However, the analysis of mean-field nucleon densities carried out in Ref. 61] by means of the Helm model, pointed out that the neutron and proton density profiles differ not only in the mean location of their surfaces but also in their surface widths.

A similar situation occurs when the NST is calculated in the DM including the surface width correction in Eq. (12). First, in Fig. 4 we display by empty symbols the NST predicted by the DM with $b_{n}=b_{p}$, for the same nuclei measured in antiprotonic atoms, computed with Eqs. (12) and (13) using the SIII and SkM* Skyrme forces and the NL-SH and NL3 relativistic mean field (RMF) parameter sets. These values are compared in Fig. 4 with the values obtained from self-consistent ETF calculations in finite nuclei 45, 62 (filled symbols). The reason for comparing DM with ETF instead of with Hartree-Fock or Hartree results, lies on the fact that both, DM and ETF, are free of shell effects that could mask the comparison. We have calculated the ETF values of the NST by using Eq. 8) with the rms radii of the self-consistent ETF neutron and proton densities of each isotope.

Two conclusions can be underlined from Fig. 4 . First, the predictions of the DM with $b_{n}=b_{p}$ systematically undershoot the ETF values of the NST computed in finite nuclei. Second, for a given nucleus the difference between the ETF value of $\Delta r_{n p}$ and the value predicted by the

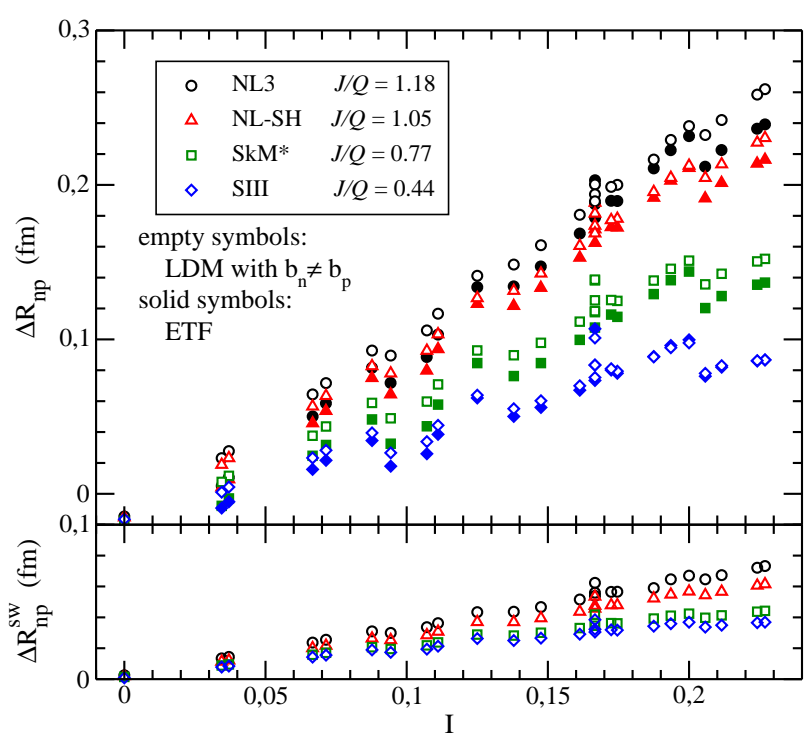

Fig. 5. (Color online) Upper panel: the same as in Fig. (4) but here the DM values include the surface width correction $\Delta r_{n p}^{s w}$ [Eq. (17)] with $b_{n}$ and $b_{p}$ obtained from ASINM as described in the text. Lower panel: surface width correction $\Delta r_{n p}^{s w}$ (the vertical scale proportionality is the same as in the upper panel). Figure taken from Ref. 31.

DM with $b_{n}=b_{p}$ is slightly larger in the RMF parameter sets than in the Skyrme forces. These facts denote that in mean-field models the surface width correction to the DM formula for the NST does not vanish, and that it has some dependence on the value of the $J / Q$ ratio.

In order to compute Eq. 12 including the surface width correction, one needs to estimate the values of the $b_{n}$ and $b_{p}$ surface widths; we proceed as before and compute $b_{n}$ and $b_{p}$ by performing ETF calculations of ASINM [30, 31, 40, 43] (see appendix of 31] for further details). Once $b_{n}$ and $b_{p}$ are known, their contribution to the NST is given by (see Eq. 12 )

$$
\Delta r_{n p}^{s w}=\sqrt{\frac{3}{5}} \frac{5}{2 R}\left(b_{n}^{2}-b_{p}^{2}\right) .
$$

The values of $\Delta r_{n p}^{s w}$ for the nuclei considered in Fig. 4 are displayed in the bottom panel of Fig. 5. We see that $\Delta r_{n p}^{s w}$ shows for each nuclear interaction an increasing linear trend with $I=(N-Z) / A$. We display the DM NST including the $\Delta r_{n p}^{s w}$ correction in the top panel of Fig. 5 by empty symbols. Note that these results correspond to adding $\Delta r_{n p}^{s w}$, shown in the bottom panel of this figure, to the DM values we have displayed in Fig. 4. We observe in the upper panel of Fig. 5 a quite good agreement between the new DM predictions and the self-consistent ETF values in finite nuclei computed with the same interaction, after including the $\Delta r_{n p}^{s w}$ correction in the DM formula.

The lower panel of Fig. 5 suggests that $\Delta r_{n p}^{s w}$ has, for each nucleus, a certain increasing trend with the $J / Q$ ratio. As discussed in more detail in Ref. 31, it is possible to fit $\Delta r_{n p}^{s w}$ by means of a law $\sigma^{s w} I$, which defines the slope $\sigma^{s w}$ of $\Delta r_{n p}^{s w}$ with respect to $I$. This slope, as 
a function of the $J / Q$ ratio for different interactions, lies inside a band limited by two straight lines, each line corresponding to the equations $\sigma^{s w}=0.3 J / Q+0.07 \mathrm{fm}$ and $\sigma^{s w}=0.3 J / Q-0.05 \mathrm{fm}$ (see Fig. 4 of Ref. [31]).

In summary, we have shown that to leading order both the bulk part $t(13)$ and the surface width term $\Delta r_{n p}^{s w}(17)$ of the DM NST are basically driven by the value of the $J / Q$ ratio. This fact suggests to fit the experimental $\Delta r_{n p}^{e x p}$ data by means of the following DM inspired ansatz:

$$
\Delta r_{n p}=\sqrt{\frac{3}{5}}\left(t-\frac{e^{2} Z}{70 J}\right)+\left(0.3 \frac{J}{Q}+c\right) I
$$

where $t$ is given by Eq. 13). The second term is the surface width correction parametrized as explained before. With the ansatz (18) we have constrained the ratio $J / Q$ by a fit to the skin data in antiprotonic atoms 53, 54. Both Eq. (18) and $t$ of Eq. (13) depend on the $J$ coefficient and the nuclear matter radius $r_{0}$. We fix them to the empirical values $J=31.6 \mathrm{MeV}$ and $r_{0}=1.143 \mathrm{fm}$ (the latter corresponds to a saturation density of $\left.0.16 \mathrm{fm}^{-3}\right)$. We consider $c=0.07 \mathrm{fm}$ and $c=-0.05 \mathrm{fm}$ in Eq. (18) to simulate the upper and lower bounds of the window of the theoretical predictions for $\sigma^{s w}$ in the nuclear mean-field models. More details of the fit can be found in Ref. 31.

The fits to experiment give $J / Q=0.667 \pm 0.047$ with $c=0.07 \mathrm{fm}$ and $J / Q=0.791 \pm 0.049$ with $c=-0.05$ fm (i.e., a total range $0.62 \leq J / Q \leq 0.84$ ). The quoted uncertainties in $J / Q$ correspond to one standard deviation associated with the fit. Both extractions of $J / Q$, for $c=$ 0.07 and $c=-0.05 \mathrm{fm}$, predict basically the same total values of the NST, which lie close 31] to the average trend $\Delta r_{n p}=(0.90 \pm 0.15) I+(-0.03 \pm 0.02) \mathrm{fm}$ [53, 54] of the experimental data. However, if one looks at the splitting of the NST into the bulk part $t$ and the surface width part $\Delta r_{n p}^{s w}$, it is different in the two cases.

As mentioned, the leading contribution to $t$ in large nuclei is the term $\frac{3}{2} r_{0}(J / Q) I$. Thus, the DM suggests that one can also expect a correlation between $\Delta r_{n p}$ and $J / Q$ in heavy nuclei. We illustrate this fact in the left panel of Fig. 6. The NST of ${ }^{208} \mathrm{~Pb}$ depicted in this figure has been obtained from self-consistent quantal calculations with Skyrme, Gogny, and RMF models 31. In the same figure the correlation between the NST in ${ }^{208} \mathrm{~Pb}$ and the slope of the symmetry energy at saturation $L$, discussed previously, is displayed to illustrate the expected $L$-vs- $J / Q$ linear correlation shown in the rightmost panel of Fig. 6. We have checked that a linear fit of $L$ against $J / Q$ depends rather weakly on the models selected to do the fit. We find that the fit lies in a range between $L=139 \mathrm{~J} / Q-52 \mathrm{MeV}$ and $L=150 \mathrm{~J} / Q-57 \mathrm{MeV}$. Considering this result and the constraint $0.6 \leq J / Q \leq 0.9$ discussed above, we find that $L$ is comprised between 31 $\mathrm{MeV}$ and $78 \mathrm{MeV}$. Therefore, our estimate (which takes into account the surface width correction in $\Delta r_{n p}$ ) for the $L$ parameter basically lies in a range $30 \leq L \leq 80 \mathrm{MeV}$.

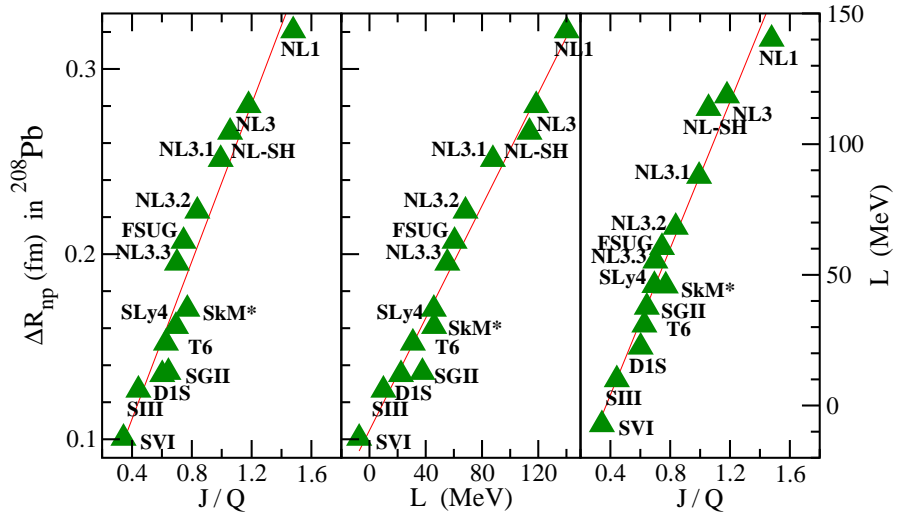

Fig. 6. (Color online) Correlation between the neutron skin thickness $\Delta r_{n p}$ in ${ }^{208} \mathrm{~Pb}$ and the ratio $J / Q$ (left panel) and between $\Delta r_{n p}$ in ${ }^{208} \mathrm{~Pb}$ and the slope of the symmetry energy at saturation $L$ (middle panel) for several mean-field models. The correlation between $L$ and the ratio $J / Q$ is also shown (right panel). The $\Delta r_{n p}$ values displayed in this figure have been computed with Eq. (8) from the rms radii of quantal self-consistent calculations with the mean-field models. Figure taken from Ref. 31.

\section{Parity-violating electron scattering and neutron skin thickness}

In the previous section we have studied the constraints on the nuclear symmetry energy that may emerge from consistently measuring the neutron skin thickness in a wide range of nuclei across the mass table with a hadronic probe. The results are very encouraging if improved accuracy could be achieved and, in our opinion, they are supportive of pursuing further systematic experimental determinations of neutron skins with hadronic probes.

In the following, we will address the complementary scenario where one explores the constraints on the nuclear symmetry energy that may be obtained from a highprecision measurement of the neutron skin thickness performed in only one single nucleus $\left({ }^{208} \mathrm{~Pb}\right.$ in our case) by using a leptonic probe.

Indeed, from Fig. 2 we see immediately that the predictions of the nuclear mean-field models for the NST of the heavy neutron-rich nucleus ${ }^{208} \mathrm{~Pb}$ vary by almost a factor of 3 , and that the slope parameter of the symmetry energy $L$ spans more than an order of magnitude between the extreme cases. In spite of such discrepancies, the mean field predictions for $\Delta r_{n p}$ and $L$ show a strong correlation (which in the previous section we have been able to understand in terms of macroscopic arguments). Thus, an accurate measurement of the NST (or of the neutron radius) of ${ }^{208} \mathrm{~Pb}$, in addition to being of fundamental importance by itself, would provide a unique constraint on the density dependence of the nuclear symmetry energy at saturation. Hence, it may be highly valuable to pursue new experiments to obtain information about the neutron distribution in a heavy nucleus as much as possible free from the uncertainties inherent in the strong interaction.

The Lead Radius Experiment (PREX) collaboration 63 65] at the Jefferson Lab has been working with the 
aforementioned purpose. PREX aims to determine the neutron radius in ${ }^{208} \mathrm{~Pb}$ to $1 \%$ accuracy by measuring the parity-violating asymmetry $\left(A_{\mathrm{pv}}\right)$ at low momentum transfer in polarized elastic electron scattering performed at a single angle. To reach a $1 \%$ accuracy in the neutron radius, the accuracy needed in $A_{\mathrm{pv}}$ has been estimated to be of a $3 \%$. The first run of PREX was successfully completed in 2010 and demonstrated the validity of this electroweak technique. The accuracy in $A_{\mathrm{pv}}$, though, was about $9 \%$ instead of $3 \%$ 65. mainly because of limited statistics in the measurement. To attain the $3 \%$-accuracy goal in $A_{\mathrm{pv}}$, a new run PREX-II will be performed [66. Recently, the motivations and optimal conditions for measuring the parity violating asymmetry in other nuclei such as ${ }^{48} \mathrm{Ca}$ and ${ }^{120} \mathrm{Sn}$ have been studied in Ref. [67] and Refs. 68, 69. Indeed, a new proposal for performing parity-violating electron scattering (PVES) on ${ }^{48} \mathrm{Ca}$ has been approved at the Jefferson Lab (CREX experiment) 70. Moreover, highprecision PVES measurements of neutron radii may become feasible in the future at the new MESA facility in Mainz 71.

For detailed informations on the PREX and CREX experiments and their physics, see the contribution to this volume by Horowitz, Kumar, and Michaels. Here, we summarize some results for completeness. The reported value for $A_{\mathrm{pv}}$ from the first run of PREX has been 65.

$$
A_{\mathrm{pv}}=0.656 \pm(0.060)_{\text {stat }} \pm(0.014)_{\text {syst }} \mathrm{ppm},
$$

at an average momentum transfer $\left\langle Q^{2}\right\rangle=0.0088 \pm 0.0001$ $\mathrm{GeV}^{2}$. Analyzed with some mild model assumptions this value of $A_{\mathrm{pv}}$ has been used to extract the following result for the neutron skin thickness in ${ }^{208} \mathrm{~Pb}$ [65]:

$$
\Delta r_{n p}=0.33_{-0.18}^{+0.16} \mathrm{fm} \text {. }
$$

A subsequent study has derived the result 72

$\Delta r_{n p}=0.302 \pm(0.175)_{\exp } \pm(0.026)_{\text {model }} \pm(0.005)_{\text {strange }} \mathrm{fm}$,

where the last uncertainty arises from the uncertainty in the electric strange quark form factor. Both results are much consistent with previous estimates, although the central value is larger than the typical value reported from the existing evidence on $\Delta r_{n p}$ of ${ }^{208} \mathrm{~Pb}$ [28]. As mentioned, a new run PREX-II has been scheduled at the Jefferson Lab 66] to improve the statistics of the measurement and reach the original $3 \%$ accuracy in $A_{\mathrm{pv}}$.

\subsection{Theoretical considerations}

Electrons can interact with the protons and neutrons of a nucleus by exchanging photons and $Z^{0}$ bosons. Whereas the photons essentially couple to protons, the $Z^{0}$ bosons mostly couple to neutrons. This is due to the fact that the proton weak charge $\left(Q_{\mathrm{W}}^{p}\right)$ is very small in comparison with the neutron weak charge $\left(Q_{\mathrm{W}}^{p}\right)$. Namely, $Q_{\mathrm{W}}^{p}=$ $1-4 \sin ^{2} \theta_{\mathrm{W}} \simeq 0.075$ (where $\theta_{\mathrm{W}}$ is the weak mixing angle) [73] and $Q_{\mathrm{W}}^{n} \simeq-1$. The situation is practically opposite to what happens with the proton and neutron electric charges. Therefore, high-energy elastic electron-nucleus scattering may test not only the electric charge distribution but also the weak charge distribution in a nucleus. The weak interaction introduces a parity-violating term in the scattering amplitude that may be isolated by measuring $A_{\mathrm{pv}}$. Indeed, Donnelly, Dubach and Sick [74] suggested almost 25 years ago that PVES on nuclei could probe the neutron distribution via the electroweak interaction.

The parity-violating asymmetry of ultra-relativistic elastically scattered electrons is defined as the following relative difference 64, 75, 77]:

$$
A_{\mathrm{pv}}=\frac{\frac{d \sigma_{+}}{d \Omega}-\frac{d \sigma_{-}}{d \Omega}}{\frac{d \sigma_{+}}{d \Omega}+\frac{d \sigma_{-}}{d \Omega}}
$$

where $d \sigma_{ \pm} / d \Omega$ are the elastic electron-nucleus differential cross sections for incident electrons with positive or negative helicity. Depending on their helicity state, electrons interact with the Coulomb $\left(V_{\mathrm{C}}\right)$ plus the weak $\left(V_{\mathrm{W}}\right)$ potential or with their difference:

$$
V_{ \pm}(r)=V_{\mathrm{C}}(r) \pm V_{\mathrm{W}}(r)
$$

where the weak potential is

$$
V_{\mathrm{W}}(r)=\frac{G_{F}}{2 \sqrt{2}} \rho_{\mathrm{W}}(r),
$$

with $G_{F}$ the Fermi constant and $\rho_{\mathrm{W}}$ the weak charge density.

We solve the scattering problem in the distorted wave Born approximation (DWBA) [33, 34, 78, for realistic calculations of the parity violating asymmetry defined in Eq. 22). That is, we perform the exact phase shift analysis of the Dirac equation [33, 78, 79.

$$
\left[\boldsymbol{\alpha} \cdot \mathbf{p}+V_{ \pm}(r)\right] \psi_{ \pm}=E \psi_{ \pm}
$$

for ultra-relativistic electrons. The electric and weak charge distributions of the studied target are the main input for solving Eq. 25). To this end, we use as a benchmark the point-like densities of protons $\left(\rho_{p}\right)$ and neutrons $\left(\rho_{n}\right)$ selfconsistently calculated with the nuclear mean-field models shown in Fig. 2, It is to be emphasized that such density distributions are at the core of the nuclear energy density functionals. Thus, in the realm of density functional theory the comparisons against experiment may always be done directly in terms of the calculated $A_{\mathrm{pv}}$.

We obtain the electric charge density distribution $\rho_{\mathrm{C}}$ of the target nucleus by folding the point-like proton and neutron densities with electromagnetic proton $\left(G_{p}\right)$ and neutron $\left(G_{n}\right)$ form factors 80 . For the weak charge density distribution $\rho_{\mathrm{W}}$, we fold the point-like densities with the electric form factors for the coupling of the $Z^{0}$ boson to the proton $\left(G_{p}^{Z^{0}}\right)$ and the neutron $\left(G_{n}^{Z^{0}}\right)$ [33, 64, 77]:

$\rho_{\mathrm{W}}(r)=\int d \mathbf{r}^{\prime}\left\{4 G_{n}^{Z^{0}}\left(r^{\prime}\right) \rho_{n}\left(\left|\mathbf{r}-\mathbf{r}^{\prime}\right|\right)+4 G_{p}^{Z^{0}}\left(r^{\prime}\right) \rho_{p}\left(\left|\mathbf{r}-\mathbf{r}^{\prime}\right|\right)\right\}$ 
with

$$
\begin{aligned}
G_{p}^{Z^{0}} & =+\frac{1}{4}\left(G_{p}-G_{n}\right)-G_{p} \sin ^{2} \theta_{\mathrm{W}} \\
G_{n}^{Z^{0}} & =-\frac{1}{4}\left(G_{p}-G_{n}\right)-G_{n} \sin ^{2} \theta_{\mathrm{W}},
\end{aligned}
$$

where we have neglected strange quark form factor contributions to the weak charge distribution 64. Substituting $G_{p}^{Z^{0}}$ and $G_{n}^{Z^{0}}$ in Eq. 26 gives

$$
\begin{aligned}
\rho_{\mathrm{W}}(r)= & Q_{\mathrm{W}}^{p} \rho_{\mathrm{C}}(r)+ \\
& Q_{\mathrm{W}}^{n} \int d \mathbf{r}^{\prime}\left\{G_{p}\left(r^{\prime}\right) \rho_{n}\left(\left|\mathbf{r}-\mathbf{r}^{\prime}\right|\right)+G_{n}\left(r^{\prime}\right) \rho_{p}\left(\left|\mathbf{r}-\mathbf{r}^{\prime}\right|\right)\right\} ;
\end{aligned}
$$

wherefore it follows that the weak charge density is dominated by the neutron density of the nucleus.

Furnstahl 4 showed that the neutron form factor of ${ }^{208} \mathrm{~Pb}$ at low momentum transfer is strongly correlated with the neutron radius $r_{n}$ of ${ }^{208} \mathrm{~Pb}$ in mean-field models. In fact, in a plane wave Born approximation (PWBA) the parity-violating asymmetry is given by $64,74,75$

$$
A_{\mathrm{pv}}^{\mathrm{PWBA}}=\frac{G_{F} q^{2}}{4 \pi \alpha \sqrt{2}}\left[4 \sin ^{2} \theta_{W}+\frac{F_{n}(q)-F_{p}(q)}{F_{p}(q)}\right]
$$

where $\alpha$ is the fine structure constant and $F_{n}(q)$ and $F_{p}(q)$ are, respectively, the neutron and proton form factors (Fourier transforms of the point neutron and proton spatial distributions). If one takes the proton form factor $F_{p}(q)$ of ${ }^{208} \mathrm{~Pb}$ at low $q$ known from experiment, the correlation between the neutron form factor and the neutron radius of ${ }^{208} \mathrm{~Pb}$ [4 suggests, in view of Eq. $\sqrt{30}$, a correlation between the parity violating asymmetry and the neutron radius of this nucleus. The latter correlation has been shown in Ref. 67 in exact DWBA calculations.

Moreover, the fact that the form factors behave as $F(q) \rightarrow 1-q^{2}\left\langle r^{2}\right\rangle / 6$ when $q \rightarrow 0$ suggests also a correlation between the parity violating asymmetry and the neutron skin thickness of the target nucleus in the lowmomentum transfer regime, since for $q \rightarrow 0$ one has

$$
A_{\mathrm{pv}}^{\mathrm{PWBA}}=\frac{G_{F} q^{2}}{4 \pi \alpha \sqrt{2}}\left[4 \sin ^{2} \theta_{W}-\Delta r_{n p}\left(r_{n}+r_{p}\right) \frac{q^{2}}{6}+\cdots\right] .
$$

Here we have written $r_{n}^{2}-r_{p}^{2}$ as $\Delta r_{n p}\left(r_{n}+r_{p}\right)$. For realistic results and comparisons against experiment, however, one cannot use the simple and insightful PWBA and full DWBA calculations have to be performed. Note that the Coulomb distortions can correct the PWBA results by more than $40 \%$ of the value found in the PWBA.

\subsection{Discussion of results for the parity violating asymmetry in ${ }^{208} \mathrm{~Pb}$}

We display in Fig. 7 the relation between the parity violating asymmetry $A_{\mathrm{pv}}$ and the neutron skin thickness $\Delta r_{n p}$

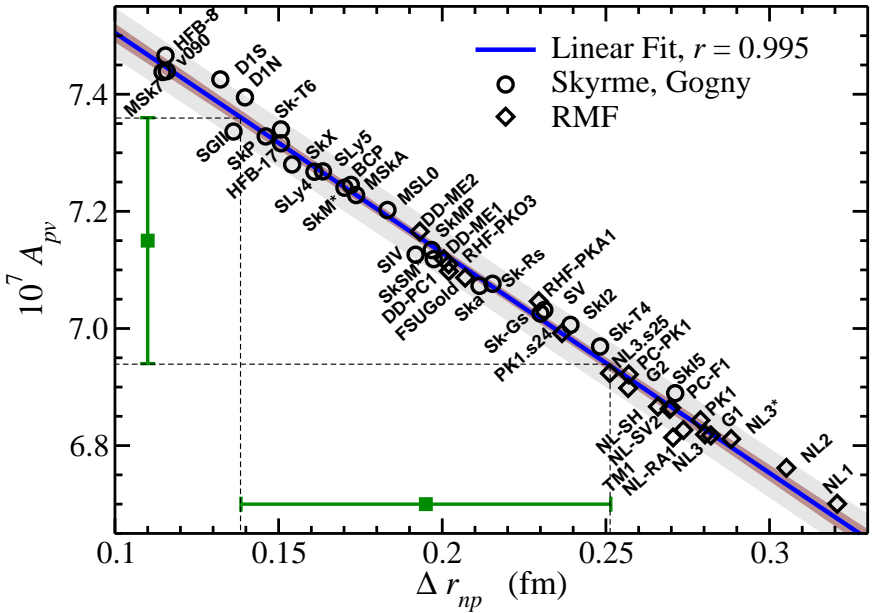

Fig. 7. (Color online) Predictions of nuclear mean-field models for the parity violating asymmetry as a function of the neutron skin thickness in ${ }^{208} \mathrm{~Pb}$. The results have a linear correlation coefficient $r=0.995$. A linear fit is shown yielding $A_{\mathrm{PV}}(\mathrm{ppm})=0.788-0.375 \Delta r_{n p}(\mathrm{fm})$. For the purpose of illustration of the accuracy in $\Delta r_{n p}$ that a $3 \%$-accuracy value of $A_{\mathrm{pv}}$ would imply, an arbitrarily chosen central point for $A_{\mathrm{pv}}$ with $3 \%$ error bars is plotted. The thinner and thicker shadowed regions represent, respectively, the $95 \%$-confidence band and 95\%-prediction band of the linear regression (see text). Figure adapted from Ref. 34.

in ${ }^{208} \mathrm{~Pb}$ within the realm of nuclear energy density functionals [34. The scattering problem has been solved in exact DWBA calculations for electrons that scatter from lead at an energy of $1.06 \mathrm{GeV}$ and an angle of $5^{\circ}$, which is close to the operating conditions of PREX.

The corpus of 47 nuclear models considered in Fig. 7 is representative of models of very different kind. We have used non-relativistic Skyrme forces (all models of Fig. 7 that start by $\mathrm{S}$ and the models HFB-8, HFB-17, v090, MSk7, MSkA, and MSL0), Gogny forces (models D1S and $\mathrm{D} 1 \mathrm{~N}$ ), and the finite-range BCP functional. On the other hand, we have considered relativistic mean-field models based on effective Lagrangians. In this case, in Fig. 7 we have included RMF models with non-linear meson couplings (these are the models with names starting by NL or PK, plus the models FSUGold, G1, G2, and TM1), meson-exchange models with density-dependent couplings (DD-ME and RHF-PK models), and finally, zero-range point-coupling models (DD-PC1, PC-PK1, and PC-PF1 models). The original references to these nuclear models can be found in the papers [20, 30, 33, 81, 82.

All of the models that we have allowed in our analysis predict the charge radius of ${ }^{208} \mathrm{~Pb}$ to better than $1 \%$ with respect to the experimental value (it is the same accuracy level as the $1 \%$ goal of PREX for the neutron radius). We have not either included models that fail significantly to predict the observed binding energy of ${ }^{208} \mathrm{~Pb}$. It would be less realistic for constraining the unknown neutron radius of ${ }^{208} \mathrm{~Pb}$ to include results for $A_{\mathrm{pv}}$ calculated with the neutron densities predicted by models that fail to describe the well-established properties of ${ }^{208} \mathrm{~Pb}$, like its charge radius 
(one has $r_{\mathrm{ch}}=5.5012(13) \mathrm{fm}$ in ${ }^{208} \mathrm{~Pb}$ [83]). Let us remark also that we have not allowed more than two models of the same kind fitted by the same group and protocol in order to minimize potential biass in our conclusions.

It can be seen from Fig. 7 that the nuclear mean-field models predict a highly linear relation between $A_{\mathrm{pv}}$ and $\Delta r_{n p}$ in ${ }^{208} \mathrm{~Pb}$, in spite of the different theoretical grounds that underly the large variety of considered models. A linear correlation coefficient $r=0.995$ is found. We also plot in Fig. 7 the 95\%-confidence band of the linear regression (colored thin inner band). The so-called confidence band represents the boundary of all of the possible straight lines fitting the results within a 95\%-confidence level, see for example Ref. 35] for details. The fact that this band is very thin in Fig. 7 is another indicator of the strong linear correlation of the results. Also displayed in Fig. 7 is the so-called prediction band 35 at a $95 \%$-confidence level (it is the wider colored band that basically coincides with the envelope of the models in the figure).

We next explore the accuracy in the determination of $\Delta r_{n p}$ that can be obtained from a $3 \%$ accuracy in $A_{\mathrm{pv}}$ (as proposed in PREX-II 66]) assuming the correlation predicted by the nuclear energy density functionals. For illustration, we have placed in Fig. 7 an arbitrary value of $A_{\mathrm{pv}}$ (viz., $0.715 \mathrm{ppm}$ ) with an attached $3 \%$ error bar (viz., $\pm 0.022 \mathrm{ppm}$ ) as depicted in the figure. Given that the correlation between $A_{\mathrm{PV}}$ and $\Delta r_{n p}$ is highly linear, the conclusions about the resulting accuracy in $\Delta r_{n p}$ will be very similar for other $A_{\mathrm{pv}}$ values, at least if they are not very far away. Even though our test central value $A_{\mathrm{pv}}=$ $0.715 \mathrm{ppm}$ is not the one measured in the first run of $\operatorname{PREX}\left(A_{\mathrm{pv}}=0.656 \pm(0.060)_{\text {stat }} \pm(0.014)_{\text {syst }} \mathrm{ppm}\right)$, they agree to within the uncertainties.

In Fig. 7, a value $A_{\mathrm{pv}}=0.715 \mathrm{ppm}$ corresponds to a point located around the central region of the theoretical predictions for $A_{\mathrm{pv}}$ in the considered models. It implies a central value for $\Delta r_{n p}$ that lies both within the range between 0.15 and $0.22 \mathrm{fm}$ suggested by the experiments with strong probes [33, 84, and within the range between 0.14 and $0.20 \mathrm{fm}$ suggested by recent constraints on the nuclear EOS derived from observed masses and radii of neutron stars 85, 86. While the latter constraints and a majority of studies point toward a fairly thin neutron skin in lead [28, it is to be mentioned that ruling out a thick neutron skin as suggested by the central value of the first run of PREX [65, 72] could be premature 87.

The $3 \%$-accuracy test value of $A_{\mathrm{pv}}$ plotted in Fig. 7 implies through the precise universal correlation displayed in the figure a neutron skin thickness $\Delta r_{n p}=0.195 \pm 0.057$ fm for ${ }^{208} \mathrm{~Pb}$. Knowing $\Delta r_{n p}$ and the proton radius of ${ }^{208} \mathrm{~Pb}$ by unfolding the finite size of the proton charge from the precisely known charge radius of this nucleus [83, 88, the neutron radius $r_{n}$ can be obtained. One can assume for $r_{n}$ the same uncertainty of $\Delta r_{n p}( \pm 0.057 \mathrm{fm}$ in our test example) because the accurate charge radius of ${ }^{208} \mathrm{~Pb} 83$ has a negligibly small uncertainty in comparison with $\Delta r_{n p}$ and $r_{n}$. Therefore, the projected accuracy in $r_{n}$ is better than $\pm 0.06 \mathrm{fm}$. This implies an accuracy of about $1 \%$ in $r_{n}$, which is in total support of the goal of the
PREX experiment. It may be underscored that the current analysis allows one to predict the NST and the neutron radius without specific assumptions about the shape of the neutron and proton density profiles.

In Ref. 34 we also studied directly the correlation between the values of $A_{\mathrm{pv}}$ and of $r_{n}$ predicted in ${ }^{208} \mathrm{~Pb}$ by the mean-field models. For the same models of Fig. 7, the calculated results of $A_{\mathrm{pv}}$ and $r_{n}$ showed a linear trend with a correlation coefficient $r=0.974$. That is, the correlation of $A_{\mathrm{pv}}$ with $r_{n}$ is a little less precise than the correlation of $A_{\mathrm{pv}}$ with $\Delta r_{n p}$. If we repeat the test that we have done before for $\Delta r_{n p}$, a $3 \%$-accuracy value of $A_{\mathrm{pv}}$ placed at $0.715 \mathrm{ppm}$ projects into a neutron radius $r_{n}$ of $5.644 \pm$ $0.065 \mathrm{fm}$ for ${ }^{208} \mathrm{~Pb}$ [34] (the experiments involving strong probes suggest a range of roughly $5.52 \mathrm{fm}$ to $5.67 \mathrm{fm}$ for the central value of $\left.r_{n}\right)$. Since the correlation of $A_{\mathrm{pv}}$ with $r_{n}$ is not as strong as the correlation of $A_{\mathrm{pv}}$ with $\Delta r_{n p}$, for the $3 \%$ accuracy assumed in $A_{\mathrm{pv}}$ a little higher uncertainty is derived from the $A_{\mathrm{pv}}$ vs $r_{n}$ correlation than from the $A_{\mathrm{pv}}$ vs $\Delta r_{n p}$ correlation (i.e, $\pm 0.065 \mathrm{fm}$ uncertainty instead of \pm 0.057 fm uncertainty, or a $14 \%$ more).

\subsection{Constraining the slope of the density dependence of the symmetry energy at saturation from PVES}

The determination of the density dependence of the nuclear symmetry energy has been identified as one of the most outstanding questions in nuclear physics for its deep implications for a wide variety of problems and phenomena. From the results discussed in the previous subsection and the fact that $\Delta r_{n p}$ of a heavy nucleus is strongly correlated with the slope $L$ of the symmetry energy at the saturation density (see Fig. 2), we can expect a linear trend of $A_{\mathrm{pv}}$ with respect to the $L$ parameter in nuclear mean-field models. This expectation is confirmed by the plot shown in Fig. 8. It implies that a measurement of the parity violating asymmetry in ${ }^{208} \mathrm{~Pb}$ directly tests the density dependence of the nuclear symmetry energy.

Note that the models with a stiffer symmetry energy around saturation density (models with larger $L$ values) have, consequently, a smaller symmetry energy at the subsaturation densities which are relevant for finite nuclei. Such models produce thicker neutron skins, and have smaller values of the parity violating asymmetry as it can be seen from the negative slope of $A_{\mathrm{pv}}$ as a function of $L$ in Fig. 8 .

The linear correlation coefficient of the results for $A_{\mathrm{pv}}$ in ${ }^{208} \mathrm{~Pb}$ and $L$ is again quite high $(r=0.971)$. From the confrontation of $A_{\mathrm{pv}}$ with $L$ we find the following linear relation between these two quantities in nuclear models:

$$
A_{\mathrm{pv}}(\mathrm{ppm})=0.750-0.00055 L(\mathrm{MeV}) \text {. }
$$

One of the main merits of an electroweak extraction of the critical parameter $L$ is that it would be mostly free from strong interaction uncertainties.

Assuming as before a 3\%-accuracy value of $A_{\mathrm{pv}}$, placed arbitrarily at $0.715 \mathrm{ppm}$, one finds $L=64 \pm 39 \mathrm{MeV}$. This means that a $3 \%$-accuracy in $A_{\mathrm{pv}}$ implies roughly a window of $\pm 40 \mathrm{MeV}$ in $L$. Though the central value of $L$ depends on the assumption of $0.715 \mathrm{ppm}$, the spread in the 


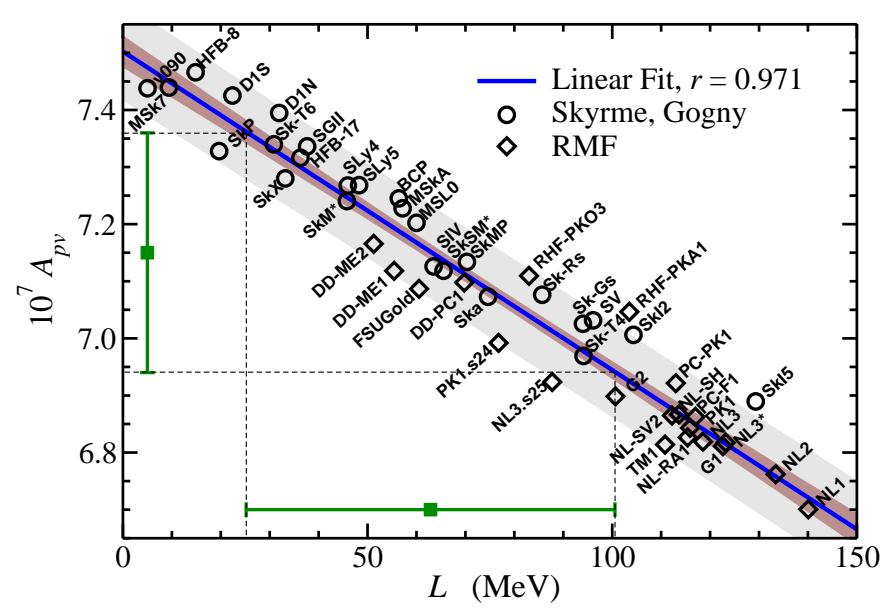

Fig. 8. (Color online) Predictions of nuclear mean-field models for the parity violating asymmetry in ${ }^{208} \mathrm{~Pb}$ as a function of the slope parameter of the nuclear symmetry energy at saturation density. The results have a linear correlation coefficient $r=0.971$. A linear fit is shown yielding $A_{\mathrm{PV}}(\mathrm{ppm})=0.750-0.00055 L(\mathrm{MeV})$. For the purpose of illustration of the accuracy in $L$ that a $3 \%$-accuracy value of $A_{\mathrm{pv}}$ would imply, an arbitrarily chosen central point for $A_{\mathrm{pv}}$ with $3 \%$ error bars is plotted. The thinner and thicker shadowed regions represent, respectively, the $95 \%$-confidence band and $95 \%$-prediction band of the linear regression (see text).

determination of $L$ associated with a 3\%-accuracy measurement of $A_{\mathrm{pv}}$ almost does not. Hence, one is bound to conclude that a $3 \%$ accuracy in the parity violating asymmetry does not impose a very narrow constraint on $L$. However, the constraint can be of great interest because PREX for the first time probes neutrons in a heavy nucleus with an electroweak probe instead of less clean strong probes.

Let us mention that the high linearity of the correlation between between $A_{\mathrm{pv}}$ and $L$ (and between $A_{\mathrm{pv}}$ and $\Delta r_{n p}$ ) found in our theoretical study shows that if $A_{\mathrm{pv}}$ could be measured with $\leq 1 \%$ accuracy, then $L$ could be estimated up to within a narrow window close to $\pm 10 \mathrm{MeV}$ accuracy (and $\Delta r_{n p}$ within a window of about $\pm 0.02 \mathrm{fm}$ ). Such a precise determination of $L$ through an electroweak measurement could have lasting impact in diverse areas of nuclear physics and astrophysics. Beyond doubt, a $\leq 1 \%$ accuracy in $A_{\mathrm{pv}}$ represents a formidable experimental challenge. Hopefully, the PREX-II experiment 66 and new facilities such as MESA in Mainz [71] will pave the way in a near future for such a goal.

\section{Size and shape of the neutron distribution in ${ }^{208} \mathbf{P b}$ and symmetry energy}

The extraction of the neutron skin thickness from experiment does not indicate in an unambiguous way whether the neutron skin is caused by an extended bulk radius, by a larger surface width of the neutron density, or by a combination of both effects 53, 59. This is a relevant aspect in experimental characterizations of neutron densities [53, 54, 89 91. It is also an interesting aspect from a theoretical point of view because in ${ }^{208} \mathrm{~Pb}$ it is related with the density dependence of the symmetry energy 33 .

The analysis of data in different types of experiments (such as electron scattering, nucleon scattering, experiments in exotic antiprotonic and pionic atoms, etc.) often involves parametrized nucleon densities. A popular profile is the two-parameter Fermi $(2 \mathrm{pF})$ function

$$
\rho(r)=\frac{\rho_{0}}{1+\exp [(r-C) / a]},
$$

where $\rho_{0}$ is central density, $C$ is the half-density radius, and $a$ is the diffuseness parameter. Albeit simple, a $2 \mathrm{pF}$ profile encodes the two most important quantities that characterize the shape of the density distributions, namely the position and thickness of the nuclear surface.

In the literature it has been found useful to distinguish two limiting situations in the formation of a neutron skin in a nucleus assuming $2 \mathrm{pF}$ shapes [53, 54, 89,91. On the one hand, in the so-called halo-type density distributions the $2 \mathrm{pF}$ nucleon profiles have $C_{n}-C_{p}=0$ and $a_{n}-a_{p}>0$. On the other hand, in the skin-type density distributions the $2 \mathrm{pF}$ nucleon profiles have $a_{n}-a_{p}=0$ and $C_{n}-C_{p}>0$.

We can analyze whether the theoretical nuclear meanfield models support either (halo or skin) type of density distributions in ${ }^{208} \mathrm{~Pb}$ by applying the strategy of Refs. [32, 33. That is, in order to obtain the predictions of the mean-field models for the central radius and diffuseness of the nucleon densities, we fit $2 \mathrm{pF}$ functions to the self-consistently calculated nucleon densities of the various models studied in the previous section. There is no unique parametrization of a given density profile with a $2 \mathrm{pF}$ function. Following Refs. [32, 33, we fit the parameters of Eq. (33) to reproduce the zeroth, second, and fourth moments of the actual self-consistent mean-field density distributions. This method has been proven to fit accurately the surface region of any realistic density profile given as an input 32,33 .

We display in Fig. 9 the difference $a_{n}-a_{p}$ between the surface diffuseness of neutrons and protons against the difference $C_{n}-C_{p}$ between the half-density radii for ${ }^{208} \mathrm{~Pb}$ in the present nuclear models. It is seen that models with a stiffer symmetry energy around saturation (i.e., models with larger values of the $L$ parameter) have larger values of the $C_{n}-C_{p}$ difference. The difference $a_{n}-a_{p}$, however, is rather insensitive to the stiffness or softness of the symmetry energy 33 .

Inspection of Fig. 9 shows that the nuclear energy density functionals overall predict density distributions with a mixed character between the halo and skin types. Nevertheless, it is to be observed that the models where the symmetry energy is softer do favor the halo-type densities, like the case of the HFB-8, MSk7, and v090 interactions. In contrast, we can see in Fig. 9 that no model predicts a skin-type distribution for ${ }^{208} \mathrm{~Pb}$, as the difference $a_{n}-a_{p}$ is in all cases systematically away from a vanishing value. It may be reminded that in Refs. [53, 54 the experimental extraction of neutron skins in antiprotonic atoms 


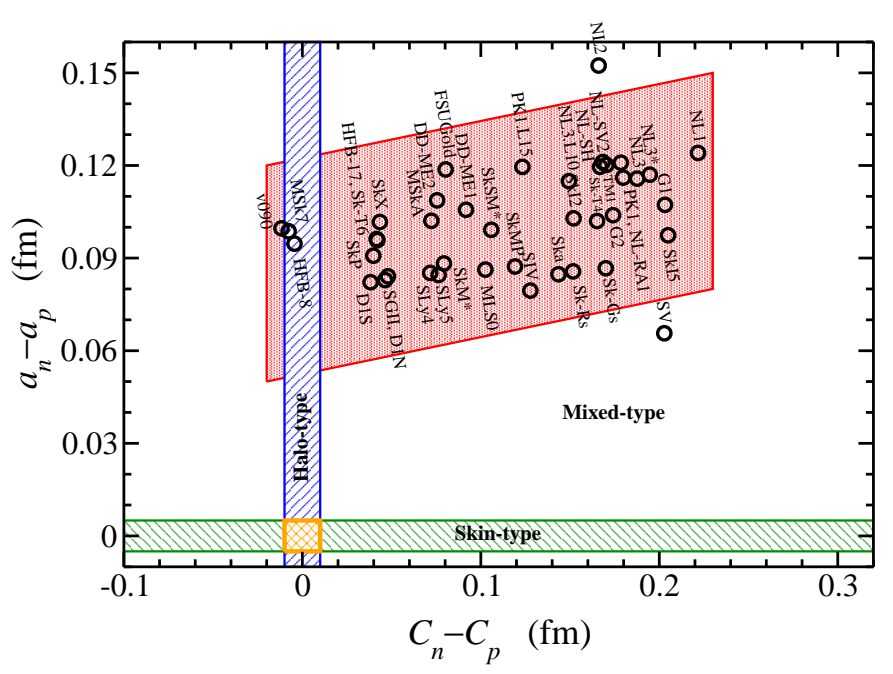

Fig. 9. (Color online) Difference $a_{n}-a_{p}$ between the surface diffuseness of the neutron and proton density profiles in ${ }^{208} \mathrm{~Pb}$ against the corresponding difference $C_{n}-C_{p}$ of the central radii as predicted by the employed mean-field models.

assumed halo-type density distributions. The theoretical predictions derived from nuclear mean-field models, thus, suggest that such an assumption is more plausible (especially if the symmetry energy is soft) than assuming that the neutron skin is formed by the mechanism of skin-type density distributions where $a_{n}-a_{p}=0$.

The specific values of the $C_{n}$ and $a_{n}$ parameters of the $2 \mathrm{pF}$ functions describing the neutron mean-field densities of ${ }^{208} \mathrm{~Pb}$ can be found in Table III of Ref. 33 for many of the nuclear interactions we have considered here. Summarizing our results, we find that the neutron half-density radius $C_{n}$ spans a range between approximately 6.6 and $7.0 \mathrm{fm}$. Meanwhile, the neutron surface diffuseness $a_{n}$ lies in a window that spans at most from 0.5 to $0.6 \mathrm{fm}$; indeed, the majority of considered nuclear mean-field models predict $a_{n}$ within 0.545 and $0.565 \mathrm{fm}$.

As mentioned in the previous section where we discussed parity-violating elastic electron scattering in ${ }^{208} \mathrm{~Pb}$, the parity violating asymmetry $A_{\mathrm{pv}}$ in the PREX experiment is obtained at one angle. Hence, assuming the electric charge distribution of ${ }^{208} \mathrm{~Pb}$ known from experiment, a determination of the shape of the neutron density of ${ }^{208} \mathrm{~Pb}$ parametrized by a $2 \mathrm{pF}$ profile requires a second measurement of $A_{\mathrm{pv}}$ at another angle [67. However, we still may get some information from a single determination of the parity violating asymmetry if we describe the neutron density profile by assuming a $2 \mathrm{pF}$ function and restrict the neutron surface diffuseness to lie within the window predicted by the nuclear mean-field models.

Working with a $2 \mathrm{pF}$ function, the neutron radius can be accurately calculated using the following formula:

$$
\left\langle r^{2}\right\rangle_{n}^{1 / 2}=\sqrt{\frac{3}{5}} C_{n}\left[1+\frac{7 \pi^{2}}{3} \frac{a_{n}^{2}}{C_{n}^{2}}\right]^{1 / 2}
$$

(see, e.g., the appendix of Ref. 32]). For any given value of the neutron radius, if we vary $a_{n}$ within a prescribed

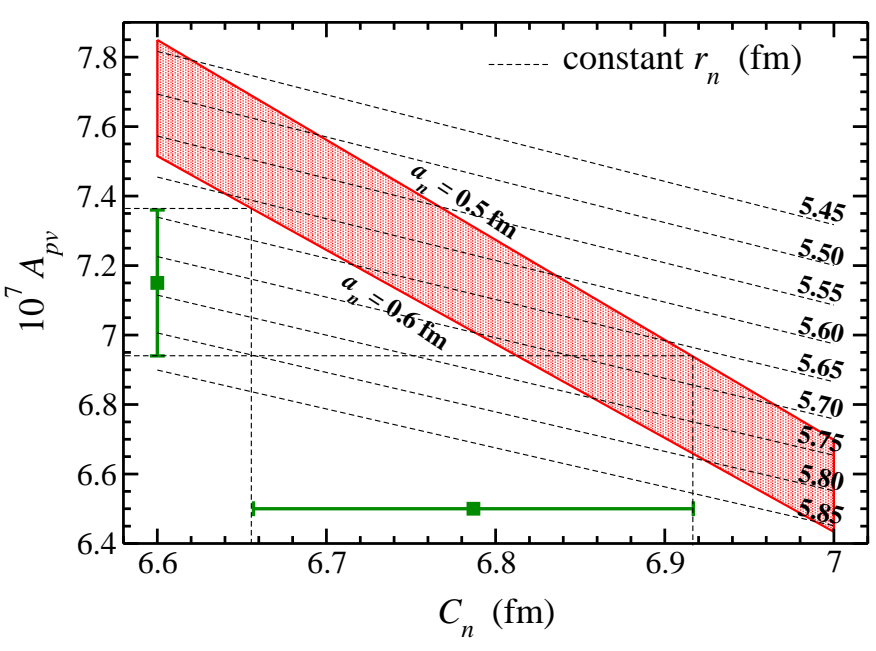

Fig. 10. (Color online) Parity violating asymmetry in ${ }^{208} \mathrm{~Pb}$ calculated using $2 \mathrm{pF}$ neutron densities and the experimental charge density of Ref. 88. The results are plotted as a function of the half-density radius $C_{n}$ of the $2 \mathrm{pF}$ profile. Lines of constant neutron radius are also drawn. The neutron surface diffuseness has been constrained to a range $0.5 \mathrm{fm} \leq a_{n} \leq 0.6$ $\mathrm{fm}$. For the purpose of illustration of the accuracy in $C_{n}$ that a $3 \%$-accuracy value of $A_{\mathrm{pv}}$ would imply, an arbitrarily chosen central point for $A_{\mathrm{pv}}$ with $3 \%$ error bars is plotted.

range of values, it will produce a family of $2 \mathrm{pF}$ functions having the same neutron radius and different central radii $C_{n}$. That is, we obtain a family of different neutron shapes having the same rms radius. We can compute the parity violating asymmetry for that family of $2 \mathrm{pF}$ neutron profiles, and then repeat the same exercise with a new value of the neutron radius.

We display in Fig. 10 the calculated parity violating asymmetry by employing the experimental charge density of Ref. 88 and $2 \mathrm{pF}$ neutron densities with the mentioned constraints. The results are plotted as a function of $C_{n}$ of the $2 \mathrm{pF}$ neutron profiles. Note that in Fig. 10 we are not using any densities of mean-field models. (For a calculation of $A_{\mathrm{pv}}$ with $2 \mathrm{pF}$ neutron and proton profiles with the parameters fitted to the theoretical mean-field densities, see section III.D of Ref. 33.) We also show in Fig. 10 the surfaces of constant neutron radius projected in the $A_{\mathrm{pv}}$-vs- $C_{n}$ plane. As expected, we find a linear correlation between $A_{\mathrm{pv}}$ and $C_{n}$ since the central radius gives the main contribution to the rms radius of a $2 \mathrm{pF}$ function in ${ }^{208} \mathrm{~Pb}$ (cf. Eq. 34 and Ref. [33]).

In analogy to our previous analysis in Section 3 , we set on top of Fig. 10 a test value of $A_{\mathrm{pv}}$ with a $3 \%$ error bar, placed at $0.715 \mathrm{ppm}$ as we did before in Section 3 . From such a test constraint and a neutron surface diffuseness $a_{n}=0.55 \pm 0.05 \mathrm{fm}$, we find a half-density radius $C_{n}=$ $6.79 \pm 0.13 \mathrm{fm}$ for the neutron density profile of ${ }^{208} \mathrm{~Pb}$. This implies a neutron rms radius $r_{n}=5.643 \pm 0.115 \mathrm{fm}$ (and a NST $\Delta r_{n p}=0.201 \pm 0.115 \mathrm{fm}$ ). Therefore, a $3 \%$ uncertainty in $A_{\mathrm{pv}}$ is projected into a $2 \%$ uncertainty in the neutron radius through the present analysis with $2 \mathrm{pF}$ neutron shapes. We note that the central values of the current predictions closely coincide with the values ex- 
tracted in our analysis carried out in Section 3.2 (namely, $r_{n}=5.644 \pm 0.065 \mathrm{fm}$ and $\left.\Delta r_{n p}=0.195 \pm 0.057 \mathrm{fm}\right)$. However, the expected uncertainty is now about twice as large. This uncertainty could be narrowed down if we had not been so conservative in our estimate of $a_{n}$ and, instead of assuming $a_{n}=0.55 \pm 0.05 \mathrm{fm}$, we had taken $a_{n}$ between 0.545 and $0.565 \mathrm{fm}$ which is the prediction of a majority of mean-field models 33 .

\section{Discussion of results and comparison with other estimates of the slope of the symmetry energy at saturation}

During the last years a major effort has been made in extracting the density dependence of the symmetry energy from experimental measurements and theoretical studies of very different nature. In Fig. 1 of the Introduction we have depicted a sample of such methods and predictions for the slope parameter $L$. Here we aim at a short presentation of some of these studies and to facilitate a comparison of existing $L$ constraints (we emphasize that it would be near to impossible to mention in this section all determinations and we apologize for those studies that inadvertently may be absent below). Additional information can be found in the quoted references and in [28, 29]. For detailed accounts of several studies mentioned in the following, see the related contributions to the present volume.

On the one side, there exist sophisticated microscopic calculations of the neutron matter EOS based on chiral effective field theory that combined with observed neutron star masses and radii 86 predict $L \sim 30-60 \mathrm{MeV}$. A determination of the EOS of neutron matter from quantum Monte Carlo calculations including realistic two- and three-nucleon forces 92 is compatible with similar $L$ values. The results from microscopic Brueckner-Hartree-Fock calculations including effective three-body forces give $L=$ $66.5 \mathrm{MeV}$ 93. These values for the slope of the symmetry energy are consistent with the range around $L \sim 40-60$ $\mathrm{MeV}$ predicted by an empirical EOS constrained exclusively on the basis of astrophysical observations (namely, a heterogeneous data set of six neutron stars) [85].

Studies of heavy-ion collisions of neutron-rich nuclei at intermediate energies are powerful sources of information to constrain $L$ [12, 13. Sometimes modeled forms of the symmetry energy, such as Eq. (16), are implied to deduce $L$. Simulations of isospin diffusion data with isospin- and momentum-dependent transport models [8, 13, 57, 58, 94 have allowed to estimate $L$ in a range between 50 and 110 $\mathrm{MeV}$. Improved quantum molecular dynamics simulations describing both isospin diffusion data and double ratios of neutron and proton spectra, support a similar range of $L$ values for a symmetry energy at saturation between 28 and $34 \mathrm{MeV}$ 16. Experiments on transverse collective flows of hydrogen and helium isotopes [95, 96] favor similar values of $L$, as well as the predictions from neutron-proton emission data [15] ( $L \sim 50 \mathrm{MeV})$ and the isotopic-scaling results of Ref. [14] $(L \sim 65 \mathrm{MeV})$.
Another source of information comes from the study of binding energies, analog states, and neutron skins. The study of masses [47, 50, 60, 97, 100, leads to $L$ values between roughly 50 and $85 \mathrm{MeV}$. In particular, the new finite-range droplet model (FRDM) predicts $L=70 \pm 15$ $\mathrm{MeV}$ [99]. Similar values of $L$ as from nuclear masses are supported by studies of $\alpha$-decay energies $(L=61 \pm 22$ $\mathrm{MeV})$ [51, Fermi-energy differences in nuclei $(L=47 \pm 18$ $\mathrm{MeV}$ ) [101, and isospin effects in nuclear charge radii $(L=54 \pm 19 \mathrm{MeV})$ [102. Excitation energies of isobaric analog states [46] point to a range $L \sim 78-111 \mathrm{MeV}$. Information on neutron distributions and skins can be obtained from nucleon elastic scattering. Recently, the neutron density of lead has been measured by polarized proton elastic scattering [84] giving $\Delta r_{n p}=0.211_{-0.063}^{+0.054} \mathrm{fm}$ in ${ }^{208} \mathrm{~Pb}$, which suggests $L=30-110 \mathrm{MeV}$ through the $\Delta r_{n p}-L$ correlation (cf. Fig. 2). An estimate of the NST in many nuclei is provided by the information obtained from antiprotonic atoms [53 55] discussed in Sections 2.2 and 2.3. Using the neutron skins of 26 stable nuclei found from this technique, we predicted a range $L=30-80 \mathrm{MeV}$ for the slope of the symmetry energy. The study of existing neutron skin data in $\mathrm{Sn}$ isotopes taken in combination with constraints from heavy-ion collisions leads to $L=58 \pm 18$ $\mathrm{MeV}$ 81. Using the data on the NST in Sn isotopes and isotope binding energy differences, a range $L=45.2 \pm 10$ $\mathrm{MeV}$ has been obtained 49. Most of the mentioned results are seen to be in good agreement among them and also with the value $L=52.7 \mathrm{MeV}$ extracted from global nucleon optical potentials [103].

In order to measure neutron radii and skins, as we have discussed in the previous sections, parity-violating elastic electron scattering is a technique free from most strong interaction uncertainties [64, 74, 77]. The feasibility of this type of experiments in heavy nuclei has been recently demonstrated by the PREX collaboration [65. The first measurement 65 had a limited statistics and the analysis of the experimental data is compatible with a wide range of $L$ values (cf. Fig. 1). A better accuracy is pursued in a future experiment PREX-II [66].

Isovector giant resonances are a further experimental source of information on the symmetry energy. Indeed, in Ref. 17] the study of the Giant Dipole Resonance (GDR) in ${ }^{208} \mathrm{~Pb}$ was used to constrain the value of the symmetry energy at $\rho=0.1 \mathrm{fm}^{-3}$ to be within 23.3 and $24.9 \mathrm{MeV}$. A study on the Giant Quadrupole Resonance (GQR) in ${ }^{208} \mathrm{~Pb}$ provides a consistent constraint $S(\rho=0.1$ $\left.\mathrm{fm}^{-3}\right)=23.3 \pm 0.6 \mathrm{MeV}$ [21]. Although the nature of the low-lying dipole strength - the so-called Pygmy Dipole Resonance (PDR) - is still under debate, it has been identified by some authors as one of the observables that can shed light into a better characterization of the properties of the nuclear symmetry energy around saturation. In Ref. [18, the measured low-energy dipole response in neutron-rich $\mathrm{Sn}$ and $\mathrm{Sb}$ isotopes and available data on ${ }^{208} \mathrm{~Pb}$ allowed to derive $J=32.0 \pm 1.8 \mathrm{MeV}$ and a pressure of pure neutron matter at saturation $p_{0}=2.3 \pm 0.8$ $\mathrm{MeV} \mathrm{fm}{ }^{-3}$, implying $L=43 \pm 15 \mathrm{MeV}$. The same observable was studied in ${ }^{68} \mathrm{Ni}$ and ${ }^{132} \mathrm{Sn}$ in Ref. 20 and 
$L=64.8 \pm 15.7 \mathrm{MeV}$ was obtained. More recently, experiments using inelastic scattering of polarized protons on ${ }^{208} \mathrm{~Pb}$ at very forward angles 104 combined with previous measurements on different energy ranges have provided the complete electric dipole response in ${ }^{208} \mathrm{~Pb}$ with high accuracy. A theoretical analysis of the experimental data 105 leads to $\Delta r_{n p}=0.168 \pm 0.022 \mathrm{fm}$, which implies $L \sim 25-60 \mathrm{MeV}$ based on the strong correlation shown in Fig. 2. A more recent theoretical investigation has found a relation between the $L$ and $J$ parameters once the experimental value of the polarizability in a heavy nucleus is known. In ${ }^{208} \mathrm{~Pb}$, such a relation is $L=-146 \pm(1)_{\text {theo. }}+\left[6.11 \pm(0.18)_{\exp .} \pm(0.26)_{\text {theo. }}\right] J$. Assuming a plausible range for $J$ of $29-33 \mathrm{MeV}$ [28, 29, the constraint $L=43 \pm 16 \mathrm{MeV}$ has been obtained [106].

Concluding, all the mentioned techniques predict values of the slope of the symmetry energy at saturation $L$ lying in a range between about 20 and $130 \mathrm{MeV}$. In spite of the discrepancies in the details, the various findings from isospin-sensitive observables, including our study of neutron skins, seem to suggest a rather soft nuclear symmetry energy around saturation. Specifically, a weighted average of the different estimates on $L$ shown in Fig. 1 - neglecting the value reported in Ref. [15 since no error bar was available - suggests a rather narrow compatible window for the central value of the $L$ parameter that lies in the range $\sim 44-68 \mathrm{MeV}$, though the large uncertainties indicate a standard deviation of about $50 \mathrm{MeV}$.

\section{Summary and outlook}

We have reviewed our main findings on the density content of the symmetry energy and the neutron skin thickness $\Delta r_{n p}$ in a heavy nucleus such as ${ }^{208} \mathrm{~Pb}$ reported in earlier literature 30, 31, 33, 34. We have discussed possible extractions of such magnitudes from two experimental techniques, one involving the strong interaction and the other one involving the electro-weak interaction.

First, we have used insights from the DM $36,38,52$ to study the NST of nuclei. In the DM picture the neutron skin consists of two contributions. One of them comes from the separation between the neutron and proton mean surfaces and the other one from the fact that the surface widths of the neutron and proton densities are different. In the DM, $\Delta r_{n p}$ is basically driven by the ratio between the symmetry energy at saturation $J$ and the surface stiffness coefficient $Q$. This ratio $J / Q$ is directly related to the symmetry energy coefficient of a finite nucleus 36 38]. To relate the NST in a nucleus with the density content of the symmetry energy in the EOS, use has been made of a generic relation in mean-field models, where the DM symmetry energy coefficient in a nucleus and the symmetry energy in nuclear matter at a subsaturation density $\rho$ nearly coincide. This relation allows one to recast the expression for $\Delta r_{n p}$ of the DM in terms of the slope parameter of the symmetry energy $L$. The result provides a clear-cut insight into the correlation, first established by Brown [2, 3, between the NST in ${ }^{208} \mathrm{~Pb}$ and the slope of the symmetry energy at saturation.
The neutron skins of 26 nuclei from ${ }^{40} \mathrm{Ca}$ to ${ }^{238} \mathrm{U}$ measured in antiprotonic atoms [53, 54] are the largest set of skins extracted uniformly with the same experimental technique for stable isotopes along the periodic table. These data show an average linear behavior with $I$ [53, 54]. From the fit of the DM formula to the skin data of Refs. [53, 54, we found that the ratio $J / Q$ lies in a range of $0.6^{-}$ 0.9 and the slope of the symmetry energy $L$ is between 30 and $80 \mathrm{MeV}$. Though the neutron skins derived from antiprotonic atoms are to some extent model dependent and have for some nuclei large uncertainties, we find that the deduced estimate of the density dependence of the symmetry energy is very compatible with other analysis. In particular, it has a large overlap with the $L$ predictions from distinct experimental information such as heavy-ion collisions, proton-nucleus scattering, and giant resonances in neutron-rich nuclei. Note that the extraction of almost all of these estimates is largely ruled by the strong interaction and, therefore, model-dependent analyses are to certain extent unavoidable.

Parity-violating electron scattering has drawn considerable attention over the recent past as a possible modelindependent probe of neutron distributions. The PREX collaboration 63 65 aims to determine the neutron radius in ${ }^{208} \mathrm{~Pb}$ with $1 \%$ accuracy by measuring the parityviolating asymmetry $A_{\mathrm{pv}}$ with $3 \%$ accuracy in polarized elastic electron scattering performed on a fixed ${ }^{208} \mathrm{~Pb}$ target. See the contribution to this volume by Horowitz, Kumar, and Michaels for a detailed account of PREX (and CREX). Motivated by this landmark experiment, prior to publication of the results of the first run 65, we reported a theoretical study about the relation of $A_{\mathrm{pv}}$ with the NST of ${ }^{208} \mathrm{~Pb}$ and with the density dependence of the symmetry energy 34. Here, we reviewed our study of 34 and extended it in some aspect. We have computed $A_{\mathrm{pv}}$ in DWBA calculations in a comprehensive set of meanfield models that reproduce the experimental charge radius of ${ }^{208} \mathrm{~Pb}$ within $1 \%$ accuracy. The electric and weak charge density distributions have been obtained from the self-consistent point-like neutron and proton densities of the nuclear models folded with electromagnetic and weak charge form factors. An almost perfect linear correlation $(r>0.99)$ between $A_{\mathrm{pv}}$ and $\Delta r_{n p}$ of ${ }^{208} \mathrm{~Pb}$ is found in the nuclear mean-field models. Also a very good correlation, but a little less linear $(r \sim 0.97)$, exists between $A_{\mathrm{pv}}$ and the neutron radius. The calculations also reveal that a $3 \%$ accuracy in $A_{\mathrm{pv}}$ leads to $1 \%$ accuracy in the neutron radius, hence supporting the goal of PREX.

A $3 \%$ accuracy in $A_{\mathrm{pv}}$ was unachieved in the first run of PREX but will be within reach of PREX-II 65, 66. Here, as in Ref. 34, we discussed a 3\%-accuracy test value placed at $A_{\mathrm{pv}}=0.715 \mathrm{ppm}$. It implies a central value for $\Delta r_{n p}$ of ${ }^{208} \mathrm{~Pb}$ within the estimates obtained both from a variety of strong probes and from information from observed masses and radii of neutron stars. Although our central value of $A_{\mathrm{pv}}$ is not the value measured in the first run of $\operatorname{PREX}\left(A_{\mathrm{pv}}=0.656 \pm(0.060)_{\text {stat }} \pm(0.014)_{\text {syst }} \mathrm{ppm}\right)$, they agree within uncertainties. Our predictions about the accuracy for extracting the neutron radius, neutron skin, 
and slope of the symmetry energy if $A_{\mathrm{pv}}$ is measured to $3 \%$ accuracy, remain valid almost independently of the central value of $A_{\mathrm{pv}}$ in the analyzed ranges.

We have found a strong correlation between $A_{\mathrm{pv}}$ and the slope of the symmetry energy $L$. A relatively large spread of $\pm 40 \mathrm{MeV}$ in $L$ is predicted if the accuracy of an $A_{\mathrm{pv}}$ measurement is at the $3 \%$ level. Our study supports that an eventual measurement of $A_{\mathrm{pv}}$ with $1 \%$ accuracy would allow to constrain $\Delta r_{n p}$ of ${ }^{208} \mathrm{~Pb}$ and $L$ within narrow bounds close to $\pm 0.02 \mathrm{fm}$ and $\pm 10 \mathrm{MeV}$, respectively.

Finally, we discussed some features of the size and shape of the neutron density distribution of ${ }^{208} \mathrm{~Pb}$ according to the mean-field models. We concluded by discussing calculations of $A_{\mathrm{pv}}$ in ${ }^{208} \mathrm{~Pb}$ performed with the experimental charge density and neutron densities parametrized by $2 \mathrm{pF}$ shapes, where the neutron surface diffuseness was varied within the range predicted by the theory.

Recently, a new measurement of the electric dipole response in ${ }^{208} \mathrm{~Pb}$ has allowed to determine the electric dipole polarizability $\alpha_{D}$ in this nucleus to $3 \%$ accuracy $\left(\alpha_{D}=20.1 \pm 0.6 \mathrm{fm}^{3}\right)$ [104]; see the contribution to this volume by Tamii, von Neumann-Cosel, and Poltoratska. A skin $\Delta r_{n p}=0.156_{-0.021}^{+0.025} \mathrm{fm}$ in ${ }^{208} \mathrm{~Pb}$ was deduced [104] via the covariance analysis of Ref. [107] within a Skyrme model. In a later study using a wide class of functionals, $\Delta r_{n p}=0.168 \pm 0.022 \mathrm{fm}$ was reported from the $\alpha_{D}$ data 105]. A new study of the $\alpha_{D}$ observable [106 has been inspired by insights from the DM. That is, the DM expression for the dipole polarizability [108:

$$
\alpha_{D}^{\mathrm{DM}}=\frac{\pi e^{2}}{54} \frac{A\left\langle r^{2}\right\rangle}{J}\left(1+\frac{5}{3} \frac{9 J}{4 Q} A^{-1 / 3}\right)
$$

considered together with the DM formula for the neutron skin thickness (cf. Eqs. (12)-(13) in Section 2.1), suggests that the dipole polarizability times the symmetry energy at saturation $\left(\alpha_{D} J\right)$ is strongly correlated with $\Delta r_{n p}$ in ${ }^{208} \mathrm{~Pb}$ and with the slope of the symmetry energy $L[106$. These correlations have been confirmed to be very accurate by self-consistent calculations in a large set of nuclear functionals [106]. A similarly strong correlation has been shown between $\alpha_{D} J$ and the parity-violating asymmetry $A_{\mathrm{pv}}$ in ${ }^{208} \mathrm{~Pb}$ [106]. Hence, precise measurements of the dipole polarizability and the parity-violating asymmetry in ${ }^{208} \mathrm{~Pb}$, and in other neutron-rich heavy- and mediummass nuclei, are expected to offer trailblazing opportunities both to constrain a fundamental property of nucleon densities such as the neutron skin thickness and to scrutinize the density dependence of the symmetry energy around nuclear saturation.

\section{Acknowledgments}

Our research has been partially funded by the Spanish Consolider-Ingenio 2010 Programme CPAN CSD2007-00042 and by Grants No. FIS2011-24154 from MICINN (Spain) and FEDER, No. 2009SGR-1289 from Generalitat de Catalunya (Spain), and No. DEC-2011/01/B/ST2/03667 from National Science Centre (Poland).

\section{References}

1. A. Steiner, M. Prakash, J. Lattimer, and P. Ellis, Physics Reports 411, 325 (2005), ISSN 0370-1573, URL http://www.sciencedirect.com/ science/article/pii/S0370157305001043.

2. B. Alex Brown, Phys. Rev. Lett. 85, 5296 (2000), URL http://link.aps.org/doi/10.1103/ PhysRevLett.85.5296.

3. S. Typel and B. A. Brown, Phys. Rev. C 64, 027302 (2001), URL http://link.aps.org/doi/10.1103/ PhysRevC.64.027302.

4. R. Furnstahl, Nuclear Physics A 706, 85 (2002), ISSN 0375-9474, URL http: //www . sciencedirect. com/science/article/pii/S0375947402008679.

5. C. J. Horowitz and J. Piekarewicz, Phys. Rev. Lett. 86, 5647 (2001), URL http://link.aps.org/doi/ 10.1103/PhysRevLett.86.5647.

6. B. G. Todd-Rutel and J. Piekarewicz, Phys. Rev. Lett. 95, 122501 (2005), URL http://link.aps. org/doi/10.1103/PhysRevLett.95.122501.

7. A. E. L. Dieperink, Y. Dewulf, D. Van Neck, M. Waroquier, and V. Rodin, Phys. Rev. C 68, 064307 (2003), URL http://link.aps.org/doi/ 10.1103/PhysRevC.68.064307

8. A. W. Steiner and B.-A. Li, Phys. Rev. C 72, 041601 (2005), URL http://link.aps.org/doi/10.1103/ PhysRevC.72.041601.

9. M. K. Gaidarov, A. N. Antonov, P. Sarriguren, and E. M. de Guerra, Phys. Rev. C 85, 064319 (2012), URL http://link.aps.org/doi/10.1103/ PhysRevC.85.064319.

10. D. T. Khoa, W. von Oertzen, and A. Ogloblin, Nuclear Physics A 602, 98 (1996), ISSN 0375-9474, URL http://www.sciencedirect.com/ science/article/pii/0375947496000917.

11. T. Gaitanos, M. D. Toro, S. Typel, V. Baran, C. Fuchs, V. Greco, and H. Wolter, Nuclear Physics A 732, 24 (2004), ISSN 0375-9474, URL http://www.sciencedirect.com/science/ article/pii/S0375947403019511.

12. V. Baran, M. Colonna, V. Greco, and M. D. Toro, Physics Reports 410, 335 (2005), ISSN 0370-1573, URL http://www.sciencedirect.com/ science/article/pii/S0370157305000025

13. B.-A. Li, L.-W. Chen, and C. M. Ko, Physics Reports 464, 113 (2008), ISSN 0370-1573, URL http://www.sciencedirect.com/science/ article/pii/S0370157308001269.

14. D. V. Shetty, S. J. Yennello, and G. A. Souliotis, Phys. Rev. C 76, 024606 (2007), URL http://link. aps.org/doi/10.1103/PhysRevC.76.024606.

15. M. A. Famiano, T. Liu, W. G. Lynch, M. Mocko, A. M. Rogers, M. B. Tsang, M. S. Wallace, R. J. Charity, S. Komarov, D. G. Sarantites, et al., Phys. Rev. Lett. 97, 052701 (2006), URL http://link. aps.org/doi/10.1103/PhysRevLett.97.052701.

16. M. B. Tsang, Y. Zhang, P. Danielewicz, M. Famiano, Z. Li, W. G. Lynch, and A. W. Steiner, Phys. Rev. 
Lett. 102, 122701 (2009), URL http://link.aps . org/doi/10.1103/PhysRevLett.102.122701.

17. L. Trippa, G. Colò, and E. Vigezzi, Phys. Rev. C 77, 061304 (2008), URL http://link.aps.org/ doi/10.1103/PhysRevC.77.061304.

18. A. Klimkiewicz, N. Paar, P. Adrich, M. Fallot, K. Boretzky, T. Aumann, D. Cortina-Gil, U. D. Pramanik, T. W. Elze, H. Emling, et al. (LAND Collaboration), Phys. Rev. C 76, 051603 (2007), URL http://link.aps.org/doi/10.1103/ PhysRevC.76.051603.

19. X. Roca-Maza, G. Pozzi, M. Brenna, K. Mizuyama, and G. Colò, Phys. Rev. C 85, 024601 (2012), URL http://link.aps.org/doi/10.1103/PhysRevC. 85.024601

20. A. Carbone, G. Colò, A. Bracco, L.-G. Cao, P. F. Bortignon, F. Camera, and O. Wieland, Phys. Rev. C 81, 041301 (2010), URL http://link.aps.org/ doi/10.1103/PhysRevC.81.041301

21. X. Roca-Maza, M. Brenna, B. K. Agrawal, P. F. Bortignon, G. Colò, L.-G. Cao, N. Paar, and D. Vretenar, Phys. Rev. C 87, 034301 (2013), URL http://link.aps.org/doi/10.1103/ PhysRevC.87.034301.

22. J. Guéna, M. Lintz, and M.-A. Bouchiat, Modern Physics Letters A 20, 375 (2005), http://www.worldscientific.com/ doi/pdf/10.1142/S0217732305016853, URL http://www.worldscientific.com/doi/abs/10. 1142/S0217732305016853.

23. T. Sil, M. Centelles, X. Viñas, and J. Piekarewicz, Phys. Rev. C 71, 045502 (2005), URL http://link. aps.org/doi/10.1103/PhysRevC.71.045502.

24. J. M. Lattimer and M. Prakash, Physics Reports 442, 109 (2007), ISSN 0370-1573, ¡ce:title¿The Hans Bethe Centennial Volume 1906-2006i/ce:title , URL http://www.sciencedirect.com/science/ article/pii/S0370157307000452.

25. T. Klähn, D. Blaschke, S. Typel, E. N. E. van Dalen, A. Faessler, C. Fuchs, T. Gaitanos, H. Grigorian, A. Ho, E. E. Kolomeitsev, et al., Phys. Rev. C 74, 035802 (2006), URL http://link.aps.org/ doi/10.1103/PhysRevC.74.035802.

26. D. T. Loan, N. H. Tan, D. T. Khoa, and J. Margueron, Phys. Rev. C 83, 065809 (2011), URL http://link.aps.org/doi/10.1103/PhysRevC. 83.065809

27. M. Dutra, O. Loureno, J. S. Sá Martins, A. Delfino, J. R. Stone, and P. D. Stevenson, Phys. Rev. C 85, 035201 (2012), URL http://link.aps.org/doi/ 10.1103/PhysRevC.85.035201.

28. M. B. Tsang, J. R. Stone, F. Camera, P. Danielewicz, S. Gandolfi, K. Hebeler, C. J. Horowitz, J. Lee, W. G. Lynch, Z. Kohley, et al., Phys. Rev. C 86, 015803 (2012), URL http://link.aps.org/doi/10.1103/ PhysRevC.86.015803.

29. J. M. Lattimer and Y. Lim, The Astrophysical Journal 771, 51 (2013), URL http://stacks .iop.org/ $0004-637 X / 771 / i=1 / a=51$.
30. M. Centelles, X. Roca-Maza, X. Viñas, and M. Warda, Phys. Rev. Lett. 102, 122502 (2009), URL http://link.aps.org/doi/10. 1103/PhysRevLett.102.122502

31. M. Warda, X. Viñas, X. Roca-Maza, and M. Centelles, Phys. Rev. C 80, 024316 (2009), URL http://link.aps.org/doi/10.1103/PhysRevC. 80.024316.

32. M. Warda, X. Viñas, X. Roca-Maza, and M. Centelles, Phys. Rev. C 81, 054309 (2010), URL http://link.aps.org/doi/10.1103/PhysRevC. 81.054309

33. M. Centelles, X. Roca-Maza, X. Viñas, and M. Warda, Phys. Rev. C 82, 054314 (2010), URL http://link.aps.org/doi/10.1103/PhysRevC. 82.054314

34. X. Roca-Maza, M. Centelles, X. Viñas, and M. Warda, Phys. Rev. Lett. 106, 252501 (2011), URL http://link.aps.org/doi/10. 1103/PhysRevLett.106.252501.

35. N. R. Draper and H. Smith, Applied Regression Analysis (3rd. ed. Wiley, New York, 1998).

36. W. D. Myers and W. Swiatecki, Annals of Physics 55, 395 (1969), ISSN 0003-4916, URL http://www.sciencedirect.com/science/ article/pii/0003491669902024.

37. W. Myers and W. Swiatecki, Annals of Physics 84, 186 (1974), ISSN 0003-4916, URL http://www.sciencedirect.com/science/ article/pii/0003491674902991.

38. W. Myers, Droplet Model of Atomic Nuclei (Plenum, New York, 1977).

39. M. Farine, J. Côté, and J. M. Pearson, Phys. Rev. C 24, 303 (1981), URL http://link.aps.org/doi/ 10.1103/PhysRevC.24.303

40. M. Brack, C. Guet, and H.-B. H. kansson, Physics Reports 123, 275 (1985), ISSN 0370-1573, URL http://www.sciencedirect.com/science/ article/pii/0370157386900785

41. K. Kolehmainen, M. Prakash, J. M. Lattimer, and J. R. Treiner, Nuclear Physics A 439, 535 (1985), ISSN 0375-9474, URL http: //www . sciencedirect. com/science/article/pii/0375947485904257.

42. J. Treiner and H. Krivine, Annals of Physics 170, 406 (1986), ISSN 0003-4916, URL http://www.sciencedirect.com/science/ article/pii/0003491686900989.

43. M. Centelles, M. D. Estal, and X. V. nas, Nuclear Physics A 635, 193 (1998), ISSN 0375-9474, URL http://www.sciencedirect.com/ science/article/pii/S0375947498001675.

44. M. D. Estal, M. Centelles, and X. Vias, Nuclear Physics A 650, 443 (1999), ISSN 0375-9474, URL http://www.sciencedirect.com/science/ article/pii/S0375947499001062.

45. M. Centelles and X. V. nas, Nuclear Physics A 563, 173 (1993), ISSN 0375-9474, URL http://www.sciencedirect.com/science/ article/pii/037594749390601S. 
46. P. Danielewicz and J. Lee, Nuclear Physics A 818, 36 (2009), ISSN 0375-9474, URL http://www.sciencedirect.com/science/ article/pii/S0375947408007926.

47. M. Liu, N. Wang, Z.-X. Li, and F.-S. Zhang, Phys. Rev. C 82, 064306 (2010), URL http://link.aps. org/doi/10.1103/PhysRevC.82.064306

48. L.-W. Chen, Phys. Rev. C 83, 044308 (2011), URL http://link.aps.org/doi/10.1103/PhysRevC. 83. 044308

49. Z. Zhang and L.-W. Chen, ArXiv e-prints (2013), 1302.5327

50. J. Dong, W. Zuo, J. Gu, and U. Lombardo, Phys. Rev. C 85, 034308 (2012), URL http://link.aps . org/doi/10.1103/PhysRevC.85.034308

51. J. Dong, W. Zuo, and J. Gu, Phys. Rev. C 87, 014303 (2013), URL http://link.aps.org/doi/10.1103/ PhysRevC.87.014303.

52. W. Myers and W. Swiatecki, Nuclear Physics A 336, 267 (1980), ISSN 0375-9474, URL http://www.sciencedirect.com/science/ article/pii/0375947480906235

53. A. Trzcińska, J. Jastrzȩbski, P. Lubiński, F. J. Hartmann, R. Schmidt, T. von Egidy, and B. Kłos, Phys. Rev. Lett. 87, 082501 (2001), URL http://link. aps.org/doi/10.1103/PhysRevLett.87.082501.

54. J. Jastrzȩbski, A. Trzcińska, P. Lubiński, B. Kłos, F. J. Hartmann, T. von Egidy, and S. Wycech, International Journal of Modern Physics E 13, 343 (2004), http://www.worldscientific.com/ doi/pdf/10.1142/S0218301304002168, URL http://www.worldscientific.com/doi/abs/10. 1142/S0218301304002168.

55. B. Kłos, A. Trzcińska, J. Jastrzȩbski, T. Czosnyka, M. Kisieliński, P. Lubiński, P. Napiorkowski, L. Pieńkowski, F. J. Hartmann, B. Ketzer, et al., Phys. Rev. C 76, 014311 (2007), URL http://link. aps.org/doi/10.1103/PhysRevC.76.014311.

56. A. Krasznahorkay, H. Akimune, A. van den Berg, N. Blasi, S. Brandenburg, M. Csatlos, M. Fujiwara, J. Gulyas, M. Harakeh, M. Hunyadi, et al., Nuclear Physics A 731, 224 (2004), ISSN 0375-9474, URL http://www.sciencedirect.com/ science/article/pii/S0375947403018712.

57. L.-W. Chen, C. M. Ko, and B.-A. Li, Phys. Rev. Lett. 94, 032701 (2005), URL http://link.aps. org/doi/10.1103/PhysRevLett.94.032701.

58. L.-W. Chen, C. M. Ko, and B.-A. Li, Phys. Rev. C 72, 064309 (2005), URL http://link.aps.org/ doi/10.1103/PhysRevC.72.064309.

59. W. J. Świątecki, A. Trzcińska, and J. Jastrzȩbski, Phys. Rev. C 71, 047301 (2005), URL http: //link. aps.org/doi/10.1103/PhysRevC.71.047301.

60. P. Danielewicz, Nuclear Physics A 727, 233 (2003), ISSN 0375-9474, URL http: //www. sciencedirect. com/science/article/pii/S0375947403016622.

61. S. Mizutori, J. Dobaczewski, G. A. Lalazissis, W. Nazarewicz, and P.-G. Reinhard, Phys. Rev. C 61, 044326 (2000), URL http://link.aps.org/
doi/10.1103/PhysRevC.61.044326.

62. M. Centelles, M. Pi, X. V. nas, F. Garcias, and M. Barranco, Nuclear Physics A 510, 397 (1990), ISSN 0375-9474, URL http: //www. sciencedirect. com/science/article/pii/037594749090058T.

63. R. Michaels et al. (PREX Collaboration) (2005), URL http://hallaweb.jlab.org/parity/prex/ pbup2005.ps.

64. C. J. Horowitz, S. J. Pollock, P. A. Souder, and R. Michaels, Phys. Rev. C 63, 025501 (2001), URL http://link.aps.org/doi/10.1103/ PhysRevC.63.025501.

65. S. Abrahamyan, Z. Ahmed, H. Albataineh, K. Aniol, D. S. Armstrong, W. Armstrong, T. Averett, B. Babineau, A. Barbieri, V. Bellini, et al. (PREX Collaboration), Phys. Rev. Lett. 108, 112502 (2012), URL http://link.aps.org/doi/10.1103/ PhysRevLett.108.112502.

66. K. Paschke et al. (2012), pREX-II Proposal to Jefferson Lab., URL http://hallaweb.jlab.org/ parity/prex/prexII.pdf

67. S. Ban, C. J. Horowitz, and R. Michaels, Journal of Physics G: Nuclear and Particle Physics 39, 015104 (2012), URL http://stacks.iop.org/0954-3899/ $39 / i=1 / a=015104$.

68. J. Liu, Z. Ren, and T. Dong, Nuclear Physics A 888, 45 (2012), ISSN 0375-9474, URL http://www.sciencedirect.com/science/ article/pii/S0375947412001959.

69. J. Liu, Z. Ren, and T. Dong, Nuclear Physics A 900, 1 (2013), ISSN 0375-9474, URL http://www.sciencedirect.com/science/ article/pii/S0375947413000535.

70. S. Riordan et al. (2013), cREX Proposal to Jefferson Lab., URL http://hallaweb.jlab.org/parity/ prex/c-rex2013_v7.pdf

71. C. Sfienti (2013), see talk at the "Workshop to Explore Physics Opportunities with Intense, Polarized Electron Beams up to $300 \mathrm{MeV}$ " and "Calcium Radius Experiment (CREX) Workshop at Jefferson Lab".

72. C. J. Horowitz, Z. Ahmed, C.-M. Jen, A. Rakhman, P. A. Souder, M. M. Dalton, N. Liyanage, K. D. Paschke, K. Saenboonruang, R. Silwal, et al., Phys. Rev. C 85, 032501 (2012), URL http://link.aps. org/doi/10.1103/PhysRevC.85.032501.

73. P. J. Mohr (2011), URL http://pdg.lbl.gov/ 2012/reviews/rpp2012-rev-phys-constants . pdf.

74. T. Donnelly, J. Dubach, and I. Sick, Nuclear Physics A 503, 589 (1989), ISSN 0375-9474, URL http://www.sciencedirect.com/science/ article/pii/0375947489904326.

75. C. J. Horowitz, Phys. Rev. C 57, 3430 (1998), URL http://link.aps.org/doi/10.1103/PhysRevC. 57.3430

76. D. Vretenar, P. Finelli, A. Ventura, G. A. Lalazissis, and P. Ring, Phys. Rev. C 61, 064307 (2000), URL http://link.aps.org/doi/10.1103/ 
PhysRevC.61.064307.

77. O. Moreno, E. M. de Guerra, P. Sarriguren, and J. M. Udas, Journal of Physics G: Nuclear and Particle Physics 37, 064019 (2010), URL http://stacks. iop.org/0954-3899/37/i=6/a=064019.

78. X. Roca-Maza, M. Centelles, F. Salvat, and X. Viñas, Phys. Rev. C 78, 044332 (2008), URL http://link. aps.org/doi/10.1103/PhysRevC.78.044332.

79. F. Salvat, A. Jablonski, and C. J. Powell, Computer Physics Communications 165, 157 (2005), ISSN 0010-4655, URL http://www.sciencedirect.com/ science/article/pii/S0010465504004795.

80. J. Friedrich and T. Walcher, Eur. Phys. A 17, 607 (2003), URL http://dx.doi.org/10.1140/epja/ i $2003-10025-3$

81. L.-W. Chen, C. M. Ko, B.-A. Li, and J. Xu, Phys. Rev. C 82, 024321 (2010), URL http://link.aps. org/doi/10.1103/PhysRevC.82.024321

82. M. Baldo, P. Schuck, and X. V. nas, Physics Letters B 663, 390 (2008), ISSN 0370-2693, URL http://www.sciencedirect.com/science/ article/pii/S0370269308004383

83. I. Angeli and K. Marinova, Atomic Data and Nuclear Data Tables 99, 69 (2013), ISSN 0092-640X, URL http://www . sciencedirect .com/ science/article/pii/S0092640X12000265.

84. J. Zenihiro, H. Sakaguchi, T. Murakami, M. Yosoi, Y. Yasuda, S. Terashima, Y. Iwao, H. Takeda, M. Itoh, H. P. Yoshida, et al., Phys. Rev. C 82, 044611 (2010), URL http://link.aps.org/doi/ 10.1103/PhysRevC.82.044611.

85. A. W. Steiner, J. M. Lattimer, and E. F. Brown, The Astrophysical Journal 722, 33 (2010), URL http: //stacks. iop.org/0004-637X/722/i=1/a=33.

86. K. Hebeler, J. M. Lattimer, C. J. Pethick, and A. Schwenk, Phys. Rev. Lett. 105, 161102 (2010), URL http://link.aps.org/doi/10.1103/ PhysRevLett.105.161102.

87. F. Fattoyev and J. Piekarewicz (2013), nucl-th/ 1306.6034, URL http://arxiv.org/abs/1306. 6034

88. H. D. Vries, C. D. Jager, and C. D. Vries, Atomic Data and Nuclear Data Tables 36, 495 (1987), ISSN 0092-640X, URL http://www . sciencedirect.com/ science/article/pii/0092640X87900131.

89. E. Friedman and A. Gal, Nuclear Physics A 724,143 (2003), ISSN 0375-9474, URL http://www.sciencedirect.com/science/ article/pii/S0375947403014763

90. E. Friedman, A. Gal, and J. Mare, Nuclear Physics A 761, 283 (2005), ISSN 0375-9474, URL http://www.sciencedirect.com/science/ article/pii/S0375947405010146

91. E. Friedman, Hyperfine Interactions 193, 33 (2009), ISSN 0304-3843, URL http://dx.doi.org/10. 1007/s10751-009-0066-x.

92. S. Gandolfi, J. Carlson, and S. Reddy, Phys. Rev. C 85, 032801 (2012), URL http://link.aps.org/ doi/10.1103/PhysRevC.85.032801.
93. I. Vidaña, A. Polls, and C. m. c. Providência, Phys. Rev. C 84, 062801 (2011), URL http://link.aps. org/doi/10.1103/PhysRevC.84.062801.

94. M. B. Tsang, T. X. Liu, L. Shi, P. Danielewicz, C. K. Gelbke, X. D. Liu, W. G. Lynch, W. P. Tan, G. Verde, A. Wagner, et al., Phys. Rev. Lett. 92, 062701 (2004), URL http://link.aps.org/doi/ 10.1103/PhysRevLett.92.062701.

95. Z. Kohley, L. W. May, S. Wuenschel, A. Bonasera, K. Hagel, R. Tripathi, R. Wada, G. A. Souliotis, D. V. Shetty, S. Galanopoulos, et al., Phys. Rev. C 82, 064601 (2010), URL http://link.aps.org/ doi/10.1103/PhysRevC.82.064601.

96. Z. Kohley, L. W. May, S. Wuenschel, M. Colonna, M. Di Toro, M. Zielinska-Pfabe, K. Hagel, R. Tripathi, A. Bonasera, G. A. Souliotis, et al., Phys. Rev. C 83, 044601 (2011), URL http://link.aps.org/ doi/10.1103/PhysRevC.83.044601.

97. W. Myers and W. Swiatecki, Nuclear Physics A 601, 141 (1996), ISSN 0375-9474, URL http://www.sciencedirect.com/science/ article/pii/0375947495005099.

98. W. D. Myers and W. J. Świațecki, Phys. Rev. C 57, 3020 (1998), URL http://link.aps.org/doi/10. 1103/PhysRevC.57.3020.

99. P. Möller, W. D. Myers, H. Sagawa, and S. Yoshida, Phys. Rev. Lett. 108, 052501 (2012), URL http://link.aps.org/doi/10. 1103/PhysRevLett.108.052501.

100. B. K. Agrawal, J. N. De, and S. K. Samaddar, Phys. Rev. Lett. 109, 262501 (2012), URL http://link. aps.org/doi/10.1103/PhysRevLett.109.262501.

101. N. Wang, L. Ou, and M. Liu, Phys. Rev. C 87, 034327 (2013), URL http://link.aps.org/doi/ 10.1103/PhysRevC.87.034327.

102. N. Wang and T. Li, Phys. Rev. C 88, 011301 (2013), URL http://link.aps.org/doi/10.1103/ PhysRevC.88.011301.

103. C. Xu, B.-A. Li, and L.-W. Chen, Phys. Rev. C 82, 054607 (2010), URL http://link.aps.org/doi/ 10.1103/PhysRevC.82.054607.

104. A. Tamii et al., Phys. Rev. Lett. 107, 062502 (2011), URL http://link.aps.org/doi/10.1103/ PhysRevLett.107.062502.

105. J. Piekarewicz et al., Phys. Rev. C 85, 041302 (2012), URL http://link.aps.org/doi/10.1103/ PhysRevC.85.041302.

106. X. Roca-Maza, M. Centelles, X. Viñas, M. Brenna, G. Colò, B. K. Agrawal, N. Paar, J. Piekarewicz, and D. Vretenar (2013), 1307.4806, URL nucl-th/ http://arxiv.org/abs/1307.4806.

107. P.-G. Reinhard and W. Nazarewicz, Phys. Rev. C 81, 051303 (2010), URL http://link.aps.org/ doi/10.1103/PhysRevC.81.051303.

108. J. Meyer, P. Quentin, and B. Jennings, Nuclear Physics A 385, 269 (1982), ISSN 0375-9474, URL http://www.sciencedirect.com/science/ article/pii/0375947482901725. 